\begin{tabular}{|c|l|}
\hline Title & Three dimensional vortex structures under breaking waves \\
\hline Author(s) & Watanabe, Y asunori; Saeki, Hiroshi; Hosking, Roger J. \\
\hline Citation & Journal of Fluid Mechanics, 545, 291-328 \\
\hline https://doi.org/40.1017/S0022112005006774
\end{tabular}

Instructions for use 


\title{
Three-dimensional vortex structures under breaking waves
}

\author{
By YASUNORI WATANABE ${ }^{1}$, HIROSHI SAEKI ${ }^{1}$ \\ AND ROGER J. HOSKING ${ }^{2}$ \\ ${ }^{1}$ Division of Environment and Resources Engineering, Hokkaido University, N13 W8, \\ Sapporo 060-8628, Japan \\ ${ }^{2}$ Faculty of Science, Universiti Brunei Darussalam, Gadong BE1410, Brunei Darussalam
}

(Received 17 July 2003 and in revised form 9 May 2005)

The large-scale vortex structures under spilling and plunging breakers are investigated, using a fully three-dimensional large-eddy simulation (LES). When an overturning jet projecting from the crest in a breaking wave rebounds from the water surface ahead, the vorticity becomes unstable in a saddle region of strain between the rebounding jet and a primary spanwise vortex, resulting in spanwise undulations of the vorticity. The undulations are amplified on a braid in this saddle region, leading to a vortex loop with counter-rotating vorticity. This vortex loop consequently envelops adjacent primary vortices, to form a typical rib structure. This rib component (the stretched vortex loop) in the large-scale vortex structure, which intensifies in the strains associated with the multiple primary vortices generated throughout the splash-up cycle, appears to be the previously found obliquely descending eddy.

\section{Introduction}

When a water wave breaks, an overturning jet projects from the wave crest and plunges onto the water surface ahead of the wave, producing a rebounding jet. The rebounding jet jumps forward and plunges onto the water surface further ahead, in a resultant sequence of consecutive plunging and rebounding jets of gradually decreasing scale. This so-called splash-up cycle in a surf zone culminates in a turbulent bore, propagating towards the shore (Peregrine 1983).

The wave shape transition during the splash-up cycle was characterized by Svendsen, Madsen \& Hansen (1978). Nadaoka, Hino \& Koyano (1989) found experimentally that plunging jets produce three-dimensional coherent structures involving large-scale vortices, whereas the fully developed vortices in the turbulent bore have a much smaller scale (Svendsen \& Madsen 1984; Longo, Petti \& Losada 2002). Particular breaker types (spilling, plunging or collapsing) also have distinctive vortices in the transition region and the following bore (Battjes 1988). For instance, the smaller plunging jets in a spilling breaker consecutively spill down the front face, to form a so-called horizontal roller featuring slowly developing spanwise vortex motion (Kolaini \& Tulin 1995).

Uni-directional non-breaking waves correspond to two-dimensional irrotational flow in planes parallel to the direction of wave propagation, except in the vicinity of the water surface and the bottom. A sequence of plunging jets typically produces horizontal rollers (large-scale spanwise vortices), featuring two-dimensional rotational motion along the wave direction. The turbulent energy and Reynolds stress transition on the two-dimensional cross-section in the surf zone has been investigated 


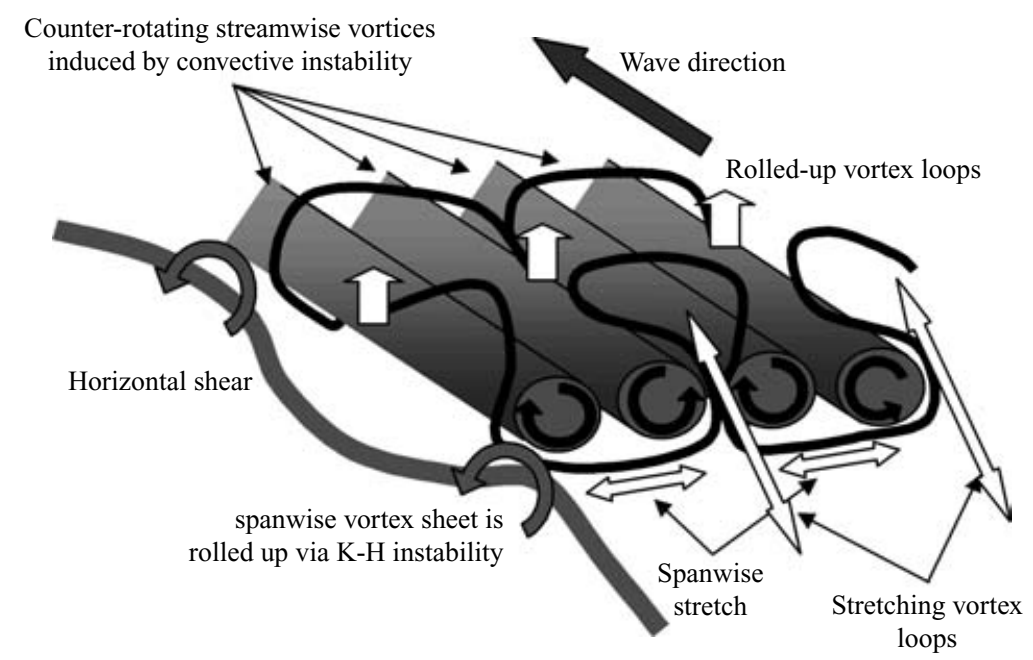

FIGURE 1. Sketch representing evolution of vortices in an internal breaking wave.

experimentally (Ting \& Kirby 1996; Cox \& Kobayashi 2000), and also by numerical simulation assuming that two-dimensional turbulence is statistically predominant (Lin \& Liu 1998; Watanabe \& Saeki 2002). Svendsen (1987) maintains that under a broken wave the spanwise (transversal) and vertical components of turbulent energy are of the same order as, but rather smaller than, the streamwise turbulent energy (in the direction of the wave propagation), and that the relative strength of the turbulent energy is analogous to that of a plane wake. When an overturning jet meets the water surface ahead, three-dimensional turbulence is initiated and intensifies in the transition region (Watanabe \& Saeki 1999). Thus the instability induced by plunging jets in a breaking wave is essentially three-dimensional, triggering the transition to unsteady turbulent flow via large-scale time-dependent three-dimensional vortex structures, as the breaking wave propagates.

The instantaneous velocity field in a surf zone typically involves an unsteady parallel shear flow towards the shore above the wave trough level, associated with the progressive bore, and an offshore flow beneath. The corresponding time-averaged velocity field is a shoreward mean flow above the trough level, and an undertow returning the water offshore. Li \& Dalrymple (1998) demonstrated that, before an incoming wave breaks, a vortex surface in the mean parallel shear flow tends to be unstable, so that periodic undulations of the vortex surface are produced. In the mean shear surface, this process is analogous to Kelvin-Helmholz $(\mathrm{K}-\mathrm{H})$ instability. They concluded that the instability induced the formation of the so-called offshore vortex train, previously observed outside the surf zone (Matsunaga, Takehara \& Awaya et al. 1988, 1994).

Instabilities occurring in stratified shear flows associated with internal waves have been studied by numerical simulation (Andreassen \& Wasberg 1994; Andreassen et al. 1998; Fritts et al. 1996, 1998). Convective instability initiates counter-rotating streamwise vortex pairs as an internal wave breaks, and K-H instability then locally intensifies a vortex sheet in parallel shears, forming spanwise vortices (cf. figure 1). These new vortices then render the previous streamwise vortices unstable via a stretchand-intensification process, which produces a complex three-dimensional vortex structure involving intertwined vortex tubes. Analogous vorticity evolution in breaking surface waves might be expected, owing to their shear flows. 
There are also several theoretical studies of the evolution of counter-rotating streamwise vortices arising between spanwise vortices consecutively arrayed in a parallel shear flow (Corcos \& Sherman 1984; Corcos \& Lin 1984; Lin \& Corcos 1984). Lasheras \& Choi (1988) experimentally studied the formations of streamwise vortices which develop from a plane free shear layer. Their interpretation is that perturbed vortex filaments placed in a stagnation region on a braid between spanwise vortices are stretched along the principal plane, and evolve into vortex loops exhibiting counter-rotating vorticity. Amplification of a perturbation at the braid then forms the three-dimensional structure in which the stretched loops envelop the spanwise vortices. A related numerical computation was carried out by Ashurst \& Meiburg (1988).

Hayakawa \& Hussain (1989) made experimental measurements for threedimensional vorticities generated behind a circular cylinder, which have a coherent structure where the so-called ribs longitudinally elongate and traverse primary vortices in turbulent wakes. Williamson (1996) discussed three-dimensional instability at a saddle point between the primary spanwise vortices shedding from a cylinder. A mechanism to form three-dimensional wakes involving vortex loops has also been found. Andreassen et al. (1998) and Fritts et al. (1998) visualize simulated coherent vortex structures in a breaking internal wave, on the basis of the definition of a vortex core developed by Jeong \& Hussain (1995). This is a good way to identify coherent vortices less dependent on the magnitude of the vorticity, and to demonstrate the evolution of the counter-rotating vortices and their interaction with the spanwise vortices produced through $\mathrm{K}-\mathrm{H}$ instability.

In the case of breaking waves, the plunging jets at first produce longitudinal arrays of spanwise vortices (referred to as horizontal eddies by Nadaoka et al. 1989), with a two-dimensional vortex structure. By tracking air bubbles under the turbulent bore, Nadaoka et al. (1989) found that an obliquely descending eddy subsequently forms, stretching downward behind the primary spanwise vortices in an inherently three-dimensional large-scale vortex structure. The axis of rotation of this obliquely descending eddy was found to coincide with the principal axis of ensemble-averaged strain behind the wave crest. Cox \& Anderson (2001) examined possible intermittent vertical rotating flows in the obliquely descending eddy and its rotation directions after a wave breaks, by particle image velocimetry (PIV) measurements. Watanabe \& Saeki (1999) investigated the vorticity evolution and the emergence of the spanwise velocity component during wave breaking using three-dimensional large-eddy simulation (LES), with a sub-grid viscosity model based on renormalization group theory (Yakhot \& Orszag 1986). Christensen \& Deigaard (2001) also used LES based on a Smagorinsky model for a breaking wave with a uniform surface in the transverse (alongshore) direction. They found that three-dimensional flow features with turbulent eddies spontaneously develop. Obliquely descending eddies were not always detected, when longitudinal vortices elongated in the wave direction appeared instead. Christensen, Walstra \& Emerat (2002) subsequently speculated that the obliquely descending eddies may be generated by a physical mechanism similar to that which produces the well-known Langmuir circulation cells owing to wind shear. Although some kind of shear instability may indeed be involved, there is of course a substantial difference in the length scale between the obliquely descending eddies and Langmuir circulation cells. Further, the shear layers which occur among the multiple large-scale primary vortices differ from wind-driven horizontal shears.

The simulations discussed above provide some insight, but the dependence of the three-dimensional structure of the vortices upon the breaker type and the initiation 
and development of the obliquely descending eddy requires further investigation. The emergence of obliquely descending eddies under certain conditions is particularly important from an engineering perspective, since they may play a prominent role in picking up and transporting bottom sediments, and hence cause pronounced localized bed and beach erosion. In this paper, we extend the work of Watanabe \& Saeki (1999) to compute the vorticity and strain fields for both plunging and spilling breakers, to investigate the three-dimensional phenomena described above. In particular, attention is focused on the initiation and evolution of the vortex structure in surf zone flow, on the basis of vortex dynamics.

We again consider gravity-dominated single-phase (liquid) flow. Air bubbles are almost inevitably present in wave breaking, so the omission of air-water interactions under the single-phase assumption does warrant some discussion. In a plunging breaker that traps air, the pressure in an initial (often large) air pocket beneath the plunging jet rapidly increases, and the cavity (the air pocket) may fragment into bubbly flow. Laboratory measurements by Lamarre \& Melville (1991) suggest that up to half of the total energy dissipation in breaking waves in deep water may go into entraining air against its buoyancy - and there can be significant bubble migration behind the jet owing to strong pressure gradients, in the initial stages of breaking. Although typical spilling breakers do not exhibit large collapsing air pockets, bubbly flow is nevertheless usually evident. A two-phase (air-water) three-dimensional model could be introduced, to investigate the extent that baroclinically generated vorticity in plunging breakers might be transported into the vortex structures produced by the progressing jets, but that is beyond the scope of this paper. However, the dynamic effects of air bubbles that commonly become entrained (in both plunging and spilling breakers) can be estimated, for the vortex structures predicted under the single-phase assumption adopted.

Aeration is discussed further in $\S 2$, following consideration of the LES numerical model used. Section 3 describes the initiation of three-dimensional instability and the resulting formation of rib structure, in the case of a plunging breaker. Section 4 is devoted to identification of the obliquely descending eddy, and the dependence of the vorticity structure on the breaker type. The results are summarized in $\S 5$. Validation of the wave-breaking model adopted is further discussed in Appendix A, and the dynamic effects of bubbles on the vortex structures in Appendix B.

\section{Numerical formulation}

As previously mentioned, the evolution of the breaking wave shape and the resulting vortex structure depends upon the breaker type. Major factors are the breaking wave height $H_{b}$, the breaking water depth $h_{b}$, the wave period $T$ (or deep-water wavelength $L_{0}$ via a dispersion relation) and the bottom slope $\theta$. A surf similarity parameter involving these factors, $\xi=\tan \theta / \sqrt{H_{b} / L_{0}}$, may be adopted to characterize the breaker type (cf. table 1). In this paper, typical plunging and spilling breakers are represented, in investigating the characteristics of surf-zone flow by large-eddy simulation (LES).

\subsection{Numerical model}

Details of the LES model may be found in Watanabe \& Saeki (1999), so we only briefly outline the model and numerical procedure here. As shown in figure 2, a Cartesian coordinate system is adopted to configure a sloping beach computing domain, where $\theta$ is a uniform angle of slope with respect to the horizontal axis. The 


\begin{tabular}{lccccccc}
\hline & $\begin{array}{c}\text { Cnoidal } \\
\text { wave } \\
\text { modulus }\end{array}$ & $\begin{array}{c}\text { Breaking } \\
\text { wave } \\
\text { height }\end{array}$ & $\begin{array}{c}\text { Breaking } \\
\text { water } \\
\text { depth }\end{array}$ & $\begin{array}{c}\text { Bottom } \\
\text { slope }\end{array}$ & $\begin{array}{c}\text { Surf } \\
\text { similarity } \\
\text { Parameter }\end{array}$ & $\begin{array}{c}\text { Wave period } \\
\text { in laboratory } \\
\text { scale (s) }\end{array}$ & $\begin{array}{c}(l, m, n) \text { grid } \\
\text { numbers } \\
\text { used }\end{array}$ \\
$\begin{array}{l}\text { type } \\
1 \text { Spilling }\end{array}$ & 0.92247 & 0.48 & 0.600 & $1 / 20$ & 0.2073 & 1.15 & $564,26,41$ \\
2 Plunging & 0.99470 & 0.58 & 0.570 & $1 / 20$ & 0.4510 & 2.75 & $564,26,41$ \\
3 Strongly & 0.99470 & 0.65 & 0.465 & $1 / 10$ & 0.8520 & 2.75 & $301,26,41$ \\
$\begin{array}{c}\text { plunging } \\
\text { Intermediate } \\
\text { (spilling- } \\
\text { plunging) }\end{array}$ & 0.97930 & 0.53 & 0.592 & $1 / 20$ & 0.2745 & 1.60 & $564,26,41$ \\
5 Plunging & 0.99740 & 0.70 & 0.624 & $1 / 20$ & 0.4433 & 2.97 & $564,26,41$
\end{tabular}

TABLE 1. Wave and numerical conditions.

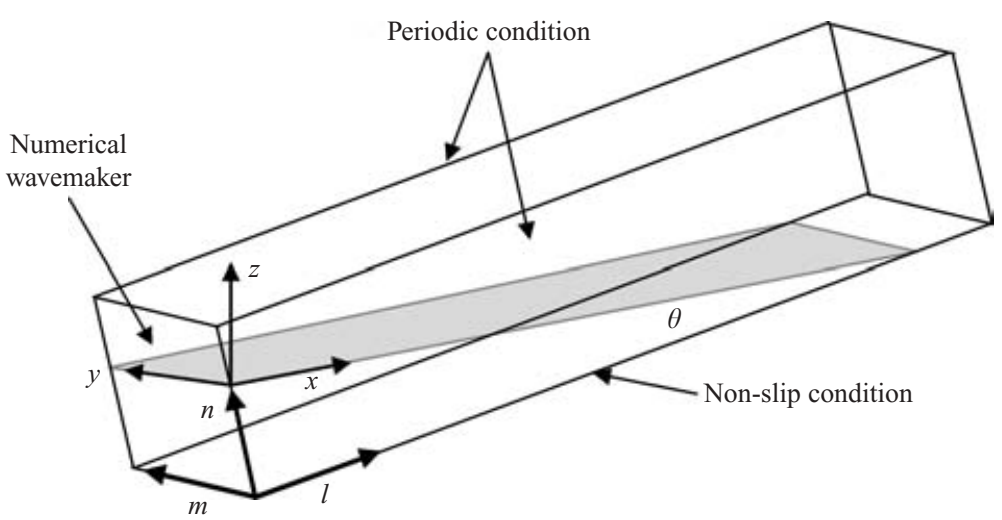

FIGURE 2. Computing domain and coordinate system.

dimensionless Navier-Stokes equation of incompressible fluid motion is

$$
\frac{\mathrm{D} \boldsymbol{u}}{\mathrm{D} t}=-\nabla p+\nabla \cdot \boldsymbol{\tau}_{\mathbf{0}}+\boldsymbol{g}
$$

where $\boldsymbol{u}$ denotes the fluid velocity, $p$ is the pressure, $\boldsymbol{\tau}_{\mathbf{0}}$ is the viscous stress tensor $\left(=2 v_{0} \boldsymbol{S}\right.$, where $v_{0}$ is the kinematic viscosity and $\boldsymbol{S}$ is the strain tensor), and $\boldsymbol{g}$ is the acceleration due to gravity. All the dimensionless variables in equation (2.1) are conveniently defined with reference to the water wave speed, the water depth at the inflow boundary, and the water density. The differential operators in equation (2.1) are the material derivative $D / D t=\partial / \partial t+u^{*} \partial / \partial l+v^{*} \partial / \partial m+w^{*} \partial / \partial n$, where $u^{*}=u \partial l / \partial x+w \partial l / \partial z, v^{*}=v$ and $w^{*}=u \partial n / \partial x+w \partial n / \partial z$, with an asterisk denoting quantities relative to the $(l, m, n)$ coordinates; the vector gradient $\nabla=((\partial l / \partial x)(\partial / \partial l)+$ $(\partial n / \partial x)(\partial / \partial n), \partial / \partial m,(\partial l / \partial z)(\partial / \partial l)+(\partial n / \partial z)(\partial / \partial n))$.

For any arbitrary variable $\psi$, the grid-scale (GS) variable $\bar{\psi}$ is obtained by the filtering operation

$$
\bar{\psi}=\int G\left(\boldsymbol{x}-\boldsymbol{x}^{\prime}\right) \psi\left(\boldsymbol{x}^{\prime}\right) \mathrm{d}^{3} \boldsymbol{x}^{\prime}
$$

where $G$ is the filter kernel, and the subgrid-scale (SGS) component is then defined as $\psi^{\prime}=\psi-\bar{\psi}$. The essential coordinate and any arbitrary variable in our sloping 
orthogonal reference system are written as

$$
\boldsymbol{x}^{*}=\boldsymbol{A} \boldsymbol{x}, \quad \boldsymbol{\psi}^{*}=\boldsymbol{A} \boldsymbol{\psi},
$$

where the orthogonal transformation matrix

$$
\boldsymbol{A}=\left[\begin{array}{ccc}
\frac{\partial l}{\partial x} & 0 & \frac{\partial l}{\partial z} \\
0 & 1 & 0 \\
\frac{\partial n}{\partial x} & 0 & \frac{\partial n}{\partial z}
\end{array}\right]
$$

so the GS variable in this transformed coordinates can be written as

$$
\overline{\psi^{*}}=\int G\left(\boldsymbol{x}^{*}-\boldsymbol{x}^{*^{\prime}}\right) \psi^{*}\left(\boldsymbol{x}^{*^{\prime}}\right) \mathrm{d}^{3} \boldsymbol{x}^{*^{\prime}}=\int G\left(\boldsymbol{A}\left(\boldsymbol{x}-\boldsymbol{x}^{\prime}\right)\right) \boldsymbol{A} \psi\left(\boldsymbol{A} \boldsymbol{x}^{\prime}\right)|\boldsymbol{A}| \mathrm{d}^{3} \boldsymbol{x}^{\prime} .
$$

Since the symmetric filter kernel $G\left(\boldsymbol{A}\left(\boldsymbol{x}-\boldsymbol{x}^{\prime}\right)\right)=G\left(\boldsymbol{x}-\boldsymbol{x}^{\prime}\right)$ and $|\boldsymbol{A}|=1$, equation (2.5) becomes

$$
\overline{\psi^{*}}=\boldsymbol{A} \bar{\psi} .
$$

Equations (2.3) and (2.6) demonstrate the invariance of the GS and SGS velocities under coordinate transformation, i.e.

$$
\overline{\boldsymbol{u}}^{*}=\boldsymbol{A} \overline{\boldsymbol{u}}, \quad \boldsymbol{u}^{*^{\prime}}=\boldsymbol{A} \boldsymbol{u}^{\prime} .
$$

After performing filtering operations, equation (2.1) becomes

$$
\frac{\mathrm{D}_{f} \overline{\boldsymbol{u}}}{\mathrm{D} t}=-\nabla \bar{p}-\nabla \cdot \boldsymbol{\tau}+\nabla \cdot \overline{\boldsymbol{\tau}_{\mathbf{0}}}+\boldsymbol{g}
$$

where $\tau$ denotes the SGS stress tensor, $\mathrm{D}_{f} / \mathrm{D} t=\partial / \partial t+\overline{u^{*}} \partial / \partial l+\overline{v^{*}} \partial / \partial m+\overline{w^{*}} \partial / \partial n$ is the filtered material derivative. The second and third terms on the right-hand side of equation (2.8) are described by the SGS viscosity form

$$
\boldsymbol{\tau}-\frac{1}{3}(\boldsymbol{\tau}: \boldsymbol{I}) \boldsymbol{I}-\overline{\boldsymbol{\tau}_{\mathbf{0}}}=-2 \nu \overline{\boldsymbol{S}}
$$

in tensor form, where $\boldsymbol{I}$ is the unit dyadic. The SGS viscosity model based on renormalization group theory (Yakhot \& Orszag 1986) is

$$
v=v_{0}\left[1+H\left(\frac{c_{s}^{2} \Delta^{4} v}{v_{0}^{3}}(2 \overline{\mathbf{S}}: \overline{\mathbf{S}})-C\right)\right]^{1 / 3},
$$

where $H$ denotes the Heaviside unit step function $(H(x)=0$ if $x \leqslant 0, H(x)=x$ if $x>0$ ), the Smagorinsky constant is $c_{s}=0.0062$, the constant $C=75$, and the filter scale $\Delta=(\Delta l \Delta m \Delta n)^{1 / 3}$ with streamwise $(\Delta l)$, spanwise $(\Delta m)$ and normal $(\Delta n)$ grid spacings. The viscosity coefficient $v$ is computed from equation (2.10) at each time step, using Newton-Raphson iteration. In passing, we note that $v$ reduces to the laminar kinematic viscosity coefficient $v_{0}$ in relatively low strain flows, and the second term on the right-hand side of (2.10) represents the effect of SGS turbulence arising in higher strain fields. Thus this SGS viscosity model is reasonable to simulate the transitional turbulence generated in breaking water waves, which were formerly described well by irrotational flow.

Alternative surface boundary conditions and some two-phase turbulence model might be incorporated in the computation, if an attempt were made to include airwater interactions ignored in this paper. The omission of air-water interactions in our model might reduce 'spiky' fluid velocity fluctuations in aerated regions, but the major 
large-scale velocity variations produced by gravity-dominated jets appear otherwise satisfactorily accounted for in the single-phase representation adopted in this paper (cf. Appendix A). Air bubbles may also displace and distort vortex flows (Sridhar \& Katz 1999), but not significantly in our context (cf. Appendix B).

\subsection{Dynamic boundary conditions}

Direct numerical simulation (DNS) of free-surface turbulence shows that a thin surface layer inducing highly anisotropic vorticity is formed at a linearized surface, and hairpin-like vortex structures are produced beneath the surface (Shen et al. 1999). Subsurface coherent vortex structures composed of both quasi-streamwise and surface-attached vortex tubes also occur in fully developed turbulent flows in open channels (Nagaosa 1999). Shen \& Yue (2001) examined the applicability of an anisotropic SGS stress model to free-surface turbulent flows.

The DNS approach may be more suitable than LES for defining small-scale structures of deforming free-surface flows under certain boundary conditions, such as for sprays or capillary waves. For example, Chen et al. (1999) used DNS to compute two-dimensional deepwater interfacial breaking waves with capillary effects taken into account. Details of the jet motion and vorticity generation at the gas-liquid interface in the splash-up cycle are presented and discussed in that paper. We may also note that surface tension may be important for smaller length scales (cf. Duncan 2001; Liu \& Duncan 2003), when there can be transverse instability of an otherwise inviscid plunging jet (Longuet-Higgins 1995). Indeed, there are many terms to consider when averaging over a free surface that is strongly affected by turbulence (Brocchini \& Peregrine $2001 a, b)$.

It seems more difficult to use DNS to evaluate lengths over the broad energy spectrum in the surf zone, ranging from the Kolmogorov length scale $O\left(\left(v^{3} / \epsilon\right)^{1 / 4}\right)$ to the wavelength of the breaking wave $O\left(\sqrt{g\left(h_{b}+H_{b}\right)} T\right)$, while LES is suitable for simulating relatively large-scale flows with a small-scale turbulent model. The vortex structures dealt with in this paper have relatively large length scales, and any relatively localized highly anisotropic turbulence near the surface is left unresolved. Indeed, in our computation, zero shear and zero surface tension conditions are applied on the surface, so that constant pressure at the surface is the free-surface dynamic boundary condition to be satisfied. The discrete form of equation (2.8), for which the fractional two-step method has been performed in advance, is solved by a predictor-corrector and CIP (cubic interpolation polynomial) method. A Poisson equation for the pressure is iteratively computed by the multi-grid method, with numerical corrections made for the pressure at the adjacent grids of any free surface via an irregular star method, to ensure the constant pressure condition $p=0$ on the surface. Small-scale (less than grid spacing) disturbances of the smooth free surface assumed in our model are implicitly excluded, and for strongly disturbed water surfaces more complicated free-surface boundary conditions would be required (Brocchini \& Peregrine 2001b).

\subsection{Kinematic boundary conditions}

So-called volume-of-fluid (VOF) methods have been used extensively for free-surface representations. However, it is now well-known that the simplest types of VOF methods such as SOLA-VOF (Hirt \& Nichols 1981) produce significant errors depending on the grid size - especially on highly curved surfaces, because of the inaccuracy of low-order schemes in reconstructing the surfaces (Scardovelli \& Zaleski 1999). A higher-order representation of the complex surface shape is essential to carry out a stable computation, such as the higher-order VOF method that Chen et al. 
(1999) used to reconstruct precisely the gas-liquid interfaces with high curvature in breaking waves.

In our computation, a density function $\left(\rho_{f}\right)$ featuring a step function at cells associated with any free surface is introduced. The density function is computed from the equation

$$
\frac{\mathrm{D}_{f} \rho_{f}(\boldsymbol{x}, t)}{\mathrm{D} t}=0
$$

via the CIP method, such that a sharp surface can be defined at each time step. In particular, $\rho_{f}$ is set to be 1 in the liquid and 0 elsewhere over the entire computational domain, and the free-surface at any time is defined to be where $\rho_{f}=0.5$. Application of (2.11) at the free surface (corresponding to $\rho_{f}=0.5$ ) is the kinematic boundary condition to be satisfied - cf. also (2.13) below. By using a high-order (Hermite spline) interpolation within a cell to define any surface profile and updating in a quasi-Lagrangean way, we can more accurately reconstruct the surface. At grids near the surface in our fixed grid system, pseudo-velocities are extrapolated from inner fluid velocities to ghost cells introduced outside the fluid boundary, and the free-surface flow is treated as if it were interfacial two-phase flow composed of liquid and ghost-liquid phases. Assuming continuity of fluid velocity across the surface, the surface velocity $\left(\boldsymbol{v}_{s}\right)$ can be defined as

$$
\boldsymbol{v}_{s}=\boldsymbol{u} \cdot \boldsymbol{n}=\boldsymbol{u}_{\text {in }} \cdot \boldsymbol{n}=\boldsymbol{u}_{\text {out }} \cdot \boldsymbol{n},
$$

where $\boldsymbol{u}_{\text {in }}$ and $\boldsymbol{u}_{\text {out }}$ represent the respective velocities just inside and outside of the surface. The free-surface kinematic boundary condition (2.11) at $\rho_{f}=0.5$ is thus

$$
\frac{\partial \rho_{f}}{\partial t}+\boldsymbol{v}_{s}\left|\nabla \rho_{f}\right|=\frac{\partial \rho_{f}}{\partial t}+\boldsymbol{u}_{\text {in }} \cdot \boldsymbol{n}\left|\nabla \rho_{f}\right|=\frac{\partial \rho_{f}}{\partial t}+\boldsymbol{u}_{\text {out }} \cdot \boldsymbol{n}\left|\nabla \rho_{f}\right|=0 .
$$

Hence the appropriate update of the surface with normal velocity $\boldsymbol{v}_{s}$ can be achieved by allocating the pseudo-fluid velocity $\boldsymbol{u}_{\text {out }}$ satisfying (2.12) on the ghost cell across the surface (through normal extrapolation). A non-slip condition is imposed at the bottom (on the plane $n=0$ ), and a periodic condition on the $m$-axis such that the variable quantities are symmetric (i.e. identical on both sides of the computation domain). The velocity, pressure and density functions are given at the inflow boundary on the basis of second-order cnoidal wave theory. The numerical and wave conditions used are summarized in table 1.

\subsection{Aeration}

As has also been previously mentioned, the cavity (the air pocket) in a plunging breaker may fragment into bubbly flow. The fragmentation is to be expected because the surface tension is readily overcome by the gravity-dominated flow, unless the cavity can be maintained by its air pressure. The plunging jet proceeds faster than the bubbly flow behind it - but again as previously mentioned, there can be significant bubble migration behind the jet owing to strong pressure gradients, at least in the early stages of breaking. Some relevant observations are described below, as background to our discussion of the dynamic effects of bubbles on the vortex structures in Appendix B.

Dean \& Stokes (2002) have closely observed cavity fragmentation and subsequent bubble entrainment in a plunging breaker. The cavity that formed at the initial plunging phase quickly fragmented into bubbles, many of them above the Hinze scale, whereas bubbles smaller than the Hinze scale were formed by the jet interaction at the wave-face. Both large bubbles from the fragmented cavity and small bubbles due to the jet/wave-face interaction then became entrained in much the same region, 
at or near the primary vortex. However, the more buoyant large bubbles tended to rise and stay in the upper part of the vortex structure, while small bubbles descended and were widely distributed. Moreover, they observed that the bubble population was predominantly smaller than the Hinze scale (cf. their figure 6). The continued entrainment of the less buoyant smaller bubbles is most probably due to the radial pressure gradient within the vortex flow (cf. also Nadaoka et al. 1989; Ruetsch \& Meiburg 1993; Druzhinin \& Elghobashi 1998). Dean \& Stokes (2002) also noted that, whereas a wave may be acoustically active inside the wave crest owing to newly created bubbles when it first breaks, it then becomes acoustically quiescent because bubbles are no longer created during the much longer evolution of the bubble plume.

If an overturning jet were regarded as a free-falling water mass, in time $t$ it would fall a vertical distance $L=g t^{2} / 2$. Since the breaking wave height $\left(H_{b}\right)$ represents an appropriate initial representative vertical length scale for the plunging jet, the characteristic time interval between the earliest splash events would therefore be $\Delta t=2 \sqrt{2 H_{b} / g}$. This time interval is approximated well by $T / 8$ for all three plunging wave cases considered later in this paper, where $T$ denotes the time between successive breaking waves. (The respective ratios of $\Delta t$ to $T / 8$ for the cases 2,3 and 5 are 1.019, 1.079 and 1.020.) Thus after the first plunging event, the phase interval $T / 8$ is commonly used to characterize the splash-up process - i.e. the time interval between the arrival of the consecutive secondary jets in these three plunging wave cases is considered to be $T / 8$. Since the primary spanwise vortices are formed at every splash, $\Delta t$ is also the characteristic time during which new vortices are produced.

From extensive observations, Cox \& Shin (2003) have found that the time variation of normalized void fractions in breaking waves is approximated well by the following model, featuring initially linear growth and then quite rapid exponential decay from a maximum:

$$
\frac{\alpha}{\alpha_{\text {ave }}}=a \frac{t}{T} \exp \left(-b \frac{t}{T}\right),
$$

where $a=800$ and $b=90$ (above still water) or 100 (below still water) are empirical coefficients. The maximum void fraction thus typically occurs at time $T / b$ after a breaking wave arrives, which is also the time-scale of the exponential decay. This early maximum aeration and equally rapid decay of the void fraction (degassing) defined by the empirical result (2.14) implies that the aeration delivered by a breaking wave is very short-lived, relative to the time-scale $(T / 8)$ of the vortex formation predicted in this paper assuming single-phase gravity-dominated flow. However, since aeration from any source might deform and displace the evolving vortex structures, the dynamic effect of air bubbles is further assessed in Appendix B for several of the wave breaking cases considered in this paper.

\section{Formation of the three-dimensional large-scale vortex structure}

As mentioned in $\S 1$, although the fluid motion in a water wave may be essentially two-dimensional (in the wave cross-section) before it breaks, shortly after breaking the fluid motion becomes three-dimensional and involves a number of large-scale vortices. At every stage where a plunging jet in the sequence meets the water ahead, the initial primary vortex has a length scale that is approximately the size of the jet, which is much longer than our computing grid spacing. The energy of the primary vortex is also much greater than the SGS turbulence component, so that subsequently the emphasis is on GS quantities in defining the dominant large-scale flow, rather than on diffusive SGS contributions. 

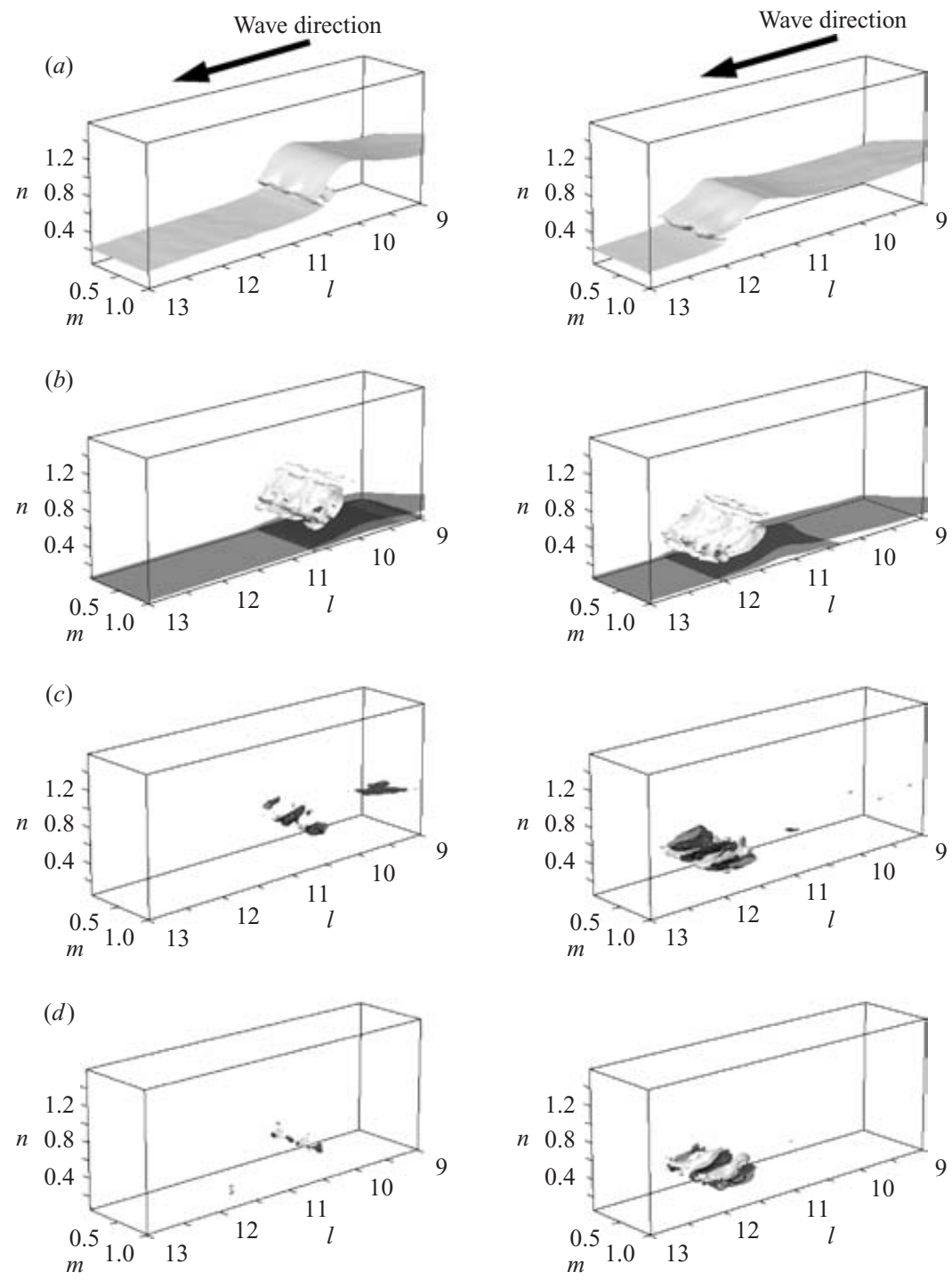

Figure 3. (a) Wave shape and simultaneous isosurfaces of $(b)$ spanwise, $(c)$ streamwise and (d) vertical vorticities at $t=t_{p}$ (left-hand column) and $t=t_{p}+T / 8$ (right-hand column) in the first wave breaking for case $2\left(t_{p}\right.$, plunging phase; $T$, wave period; white surface, +2.5 ; black surface, -2.5 ).

\subsection{Generation of the three-dimensional vorticity field}

Since a propagating periodic wavetrain is computed as an initial boundary problem from a still water state, there is no disturbed fluid until the first breaking event occurs. For the case 2 plunging breaker in table 1, figure 3 shows the surface deformation for the plunging phase in the first breaking event (when the surf zone elsewhere has undisturbed fluid), together with the isosurfaces of the spanwise, streamwise and vertical vorticities. As a spanwise vortex sheet emerges, looping along an inner surface surrounded by the overturning jet, counter-rotating streamwise and vertical vorticities simultaneously arise beneath the toe of the jet, initiating the three-dimensional 
(a)
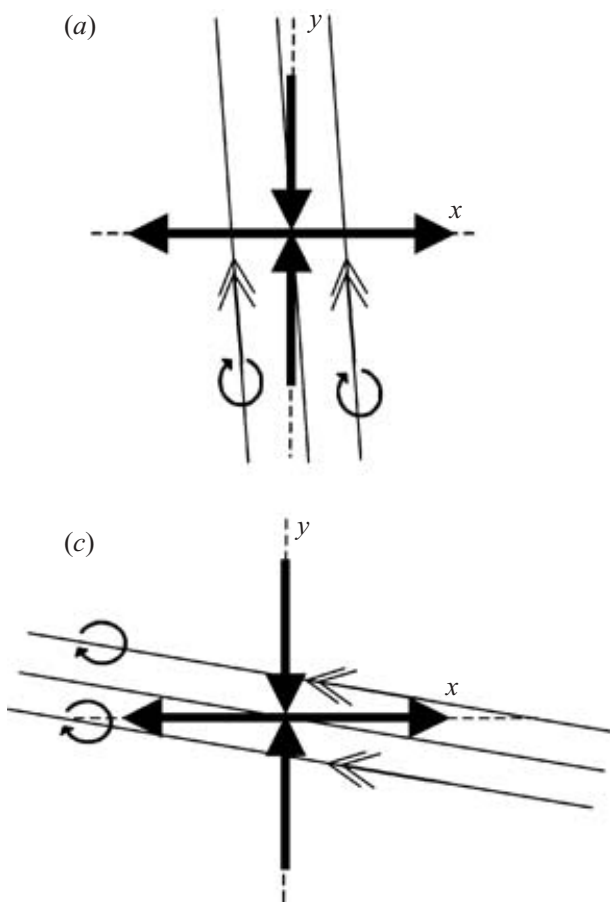

(6)

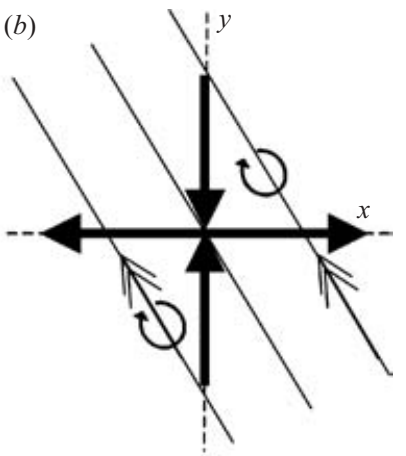

(d)
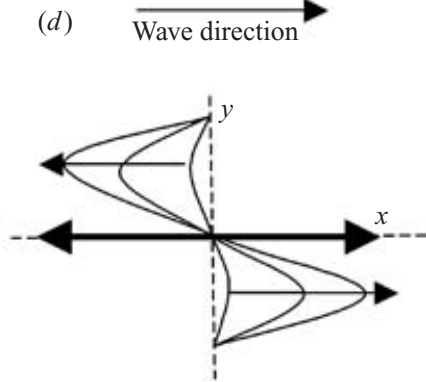

FIGURE 4. Re-orientation of the vorticity perturbations undergoing uniform stretching in the $x$-direction: (a) spanwise vortex filaments aligned at a gentle angle with the $y$-axis are stretched in the $x$-axis, $(b)$ the angle of the filaments increases as they are stretched, $(c)$ the direction of the filaments approaches the $x$-axis, and their vorticity is re-oriented in the spanwise direction, (d) the spanwise perturbations are amplified.

vorticity field. Re-orientation of perturbations of the primary vorticity owing to streamwise stretching may account for the emergence of the spanwise perturbations (cf. figure 4). Thus, the rapid growth of the spanwise perturbations, highly stretched in a saddle-point flow at the plunging point, results in the counter-rotating vortices to be discussed further in subsequent sections.

As mentioned previously, the phase interval $\delta t=T / 8$ characterizes each vortex formation. At the second plunging phase $\left(t=t_{p}+T / 8\right)$ shown in figure 3 , counterrotating vortices - in which the isosurface of streamwise and vertical vorticities exhibit a 'sausage' shape - are stretched along the trajectory of the rebounding jet projecting from the first to the second plunging point, and a coherent vortex structure develops. Consecutive local stretch in the large-scale vortex structure causes a gradual scaling down toward smaller vortices over the transition and bore region, where subsets of the vortices are dissipated and the survivors are transported by the reverse flow beneath the wave trough level up to the breaking point until the next breaking event occurs (cf. figures 5 and $8 a$ ). The remaining vorticity affects the next breaking process, when the large-scale vortices produced at that stage interact with it, forming a more complex local shear field and resultant vortex substructures with more local deformation.

Vortices in the instantaneous surf zone flow also locally alter the intensity, shape and position of the vortices involved in the coherent structure, at the onset of every wave break - cf. also figure 6 , which shows the isosurfaces of vorticity in the transition region during the third and fourth wave-breaking events for case 5 . The relative breaking wave height is greater than for case 2, and a larger vortex structure forms, 

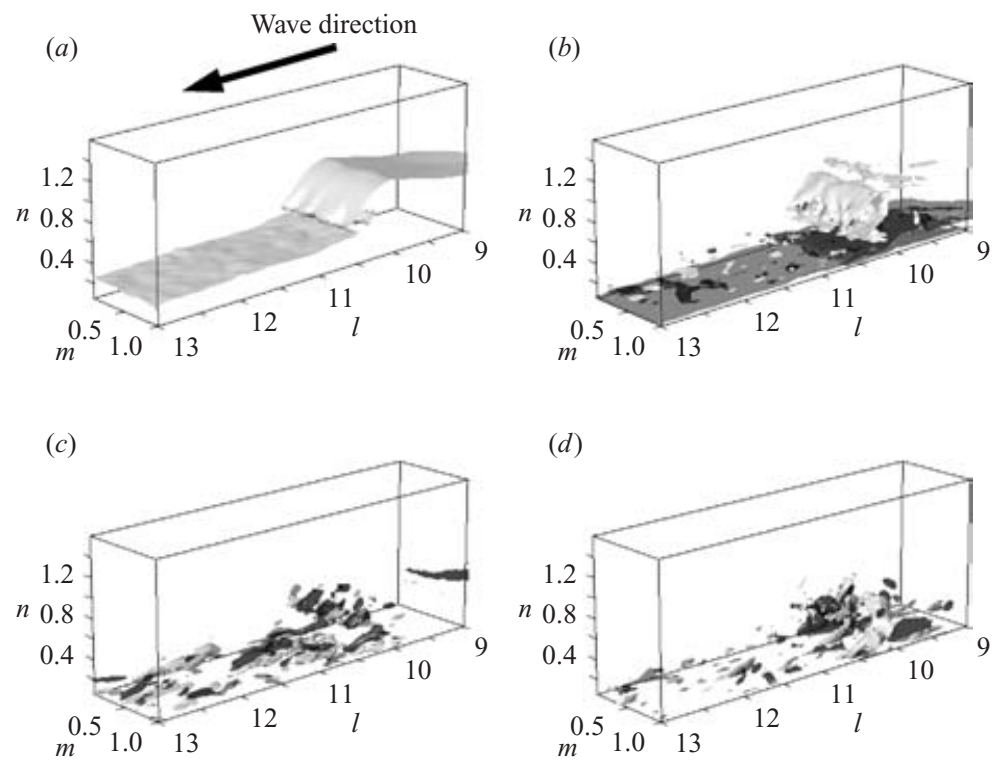

Figure 5. (a) Wave shape and simultaneous isosurfaces of $(b)$ spanwise, $(c)$ streamwise and $(d)$ vertical vorticities at the plunging phase $t=t_{p}$ in the third wave breaking for case 2 (white surface, +2.5 ; black surface, -2.5 ).

with three sets of counter-rotating vortices longitudinally traversing primary rollers of higher vorticity. This suggests that the number of vortices in the large-scale vortex structure, and the total vorticity it possesses, depends upon the breaker scale. It is notable, however, that the number of counter-rotating vortices, which derive from an inherent mode of spanwise instability, may be restricted by the number of grid points in the $m$-axis on which the periodic boundary condition is imposed. The simulated finger-like surface shape formed at the toe of the secondary jet, apparently caused by the spanwise vortex undulation corresponding to a spanwise instability mechanism as discussed below, coincides with the fingers seen in a laboratory experiment we undertook (if sprays and bubbles scattered around the jet are ignored) - cf. figure 7. This indicates that our simulation appropriately resolves the spanwise wavenumber of the instability under breaking waves on a laboratory scale.

\subsection{Shear-induced spanwise instability}

The remainder of this section explores results we obtained up to the sixth wavebreaking event, when the wave shape reaches a quasi-steady state.

Figure 8 shows the vertical ( $z$-axis), horizontal ( $x$-axis) and spanwise ( $y$-axis) distributions of the instantaneous fluid velocity in the transition region at the third plunging phase for case 2. The shoreward flow in a breaking wave-front above the trough level is found to form a horizontal parallel shear (cf. figure $8 a$ ). In figure $8 b$, the horizontal fluctuations of the vertical velocity associated with the primary vortices can be seen at each plunging point. In particular, a high vertical shear flow is formed at the third plunging point, where the plunging jet begins to rebound upward because of the sudden change in the motion of the jet. The predominant two-dimensional velocity simultaneously becomes unstable in the shear, leading to spanwise undulations of the velocity components at the plunging point (cf. figure $8 c$ ). 

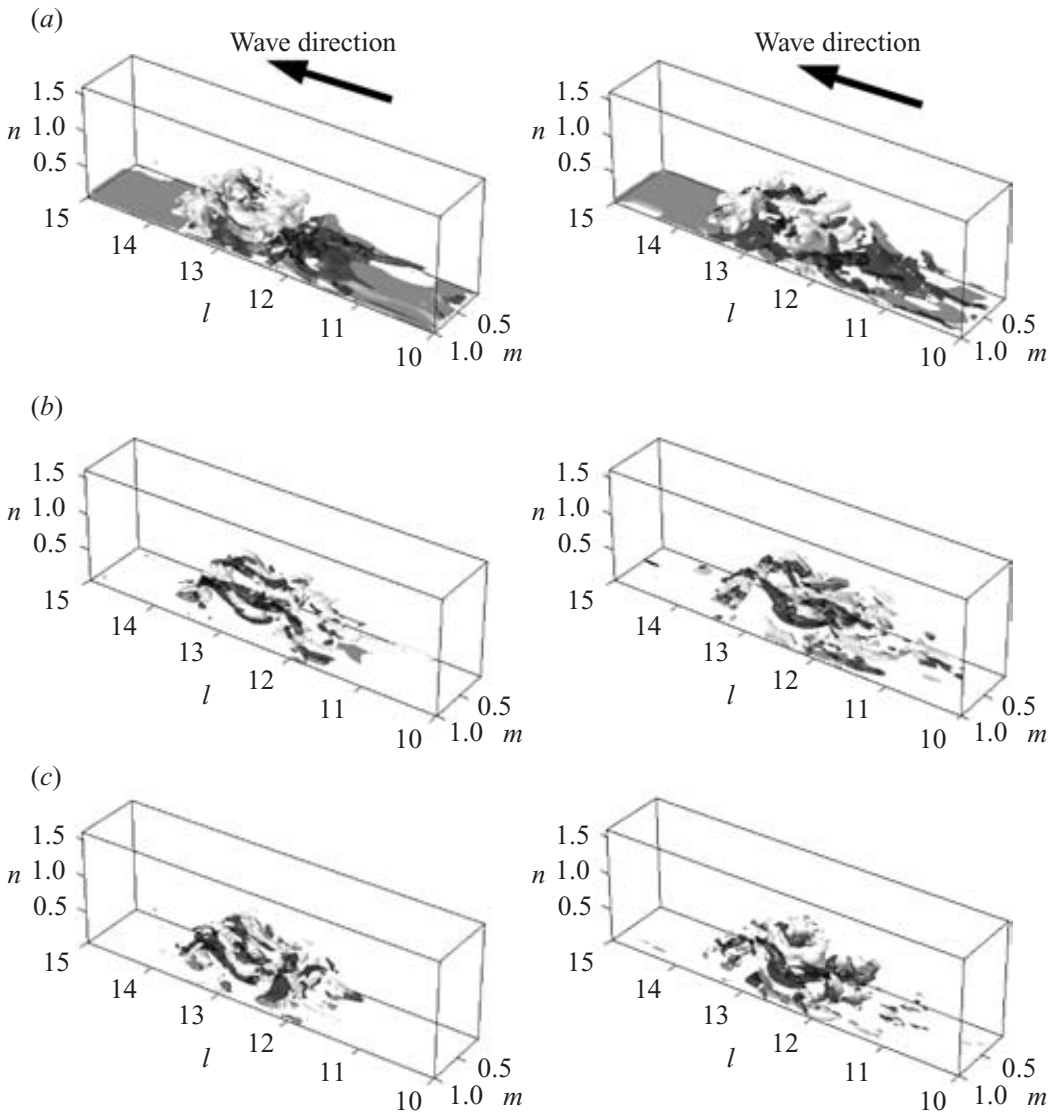

FiguRE 6. Isosurfaces of $(a)$ spanwise, $(b)$ streamwise and $(c)$ vertical vorticities in a transition region at $t=t_{p}+T / 8$ in the third wave breaking (left-hand column) and at the same phase in the fourth wave breaking (right-hand column) for case 5 (white surface, +2.5 ; black surface, $-2.5)$.

According to Andreassen et al. (1998), at the onset of internal wave breaking in stratified flow, a convective instability causes the formation of streamwise counterrotating vortex pairs, resulting in dynamical development of the three-dimensional vortex structure through shear stretch-and-intensification and interaction with the spanwise vortices arising due to $\mathrm{K}-\mathrm{H}$ instability (cf. figure 1). Although the instability triggering the transition to three-dimensional flow when a surface wave breaks may differ from that of a breaking internal wave, the evolution of the large-scale vortex structure in the surf zone appears to be associated with a local shear instability analogous to $\mathrm{K}-\mathrm{H}$ instability.

The interconnected relationship between instability, the shear flow and the vortices at the plunging point is notable. Figure 9 shows the streamlines on the centre plane of the domain $(m=0.5)$, at the second and third plunging phases. A saddle-point flow appears between the upward flow associated with the rebounding jet motion and the downward rotational flow of the primary vortex, behind the third plunging point (circled). The counter-rotating vortices and the spanwise undulation of velocity (cf. figures 3 and $8 c$ ) appear near this region, which suggests that strong stretching associated with the saddle-point flow is responsible for amplifying spanwise vorticity perturbations on the shear layer, as mentioned in $\$ 3.1$ (cf. figure 4 and also figure 10). 
(a)
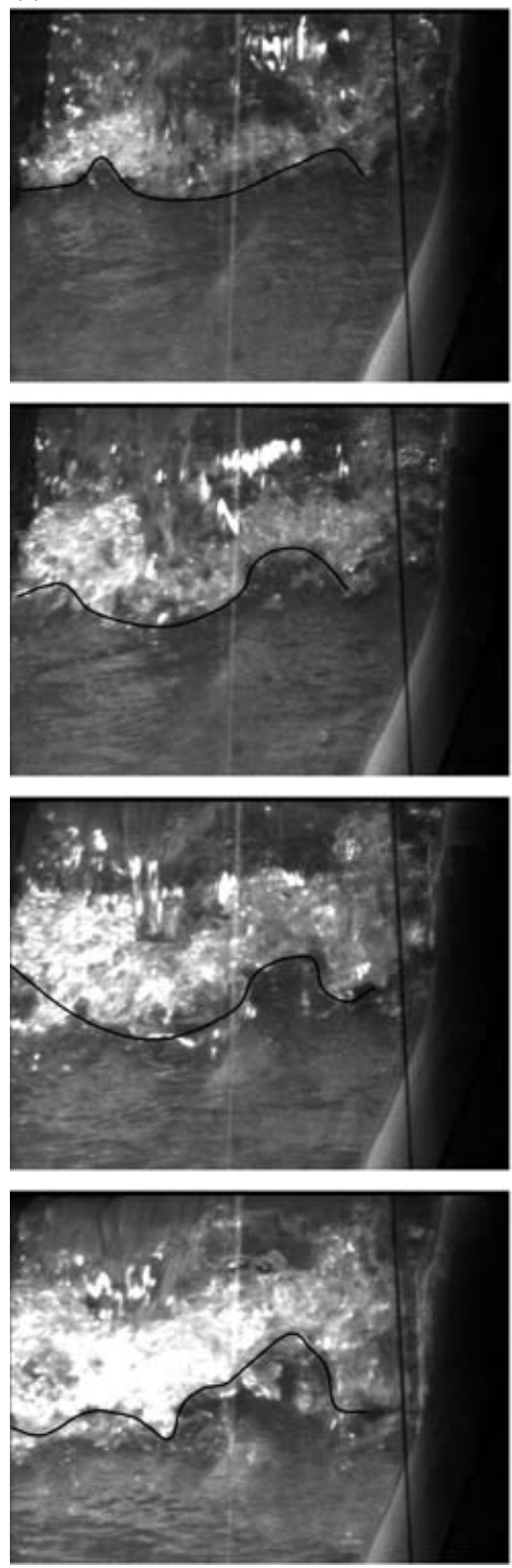

(b)
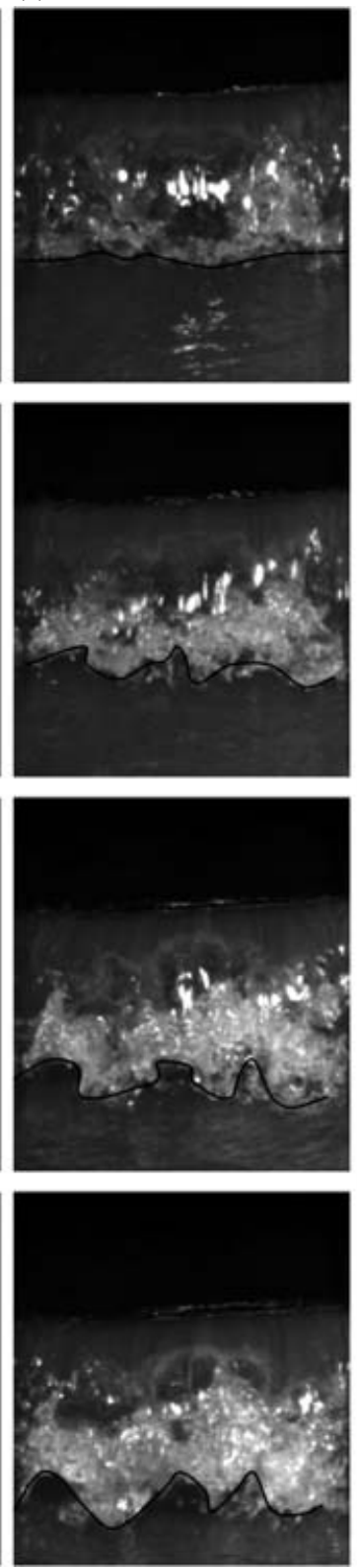

(c)

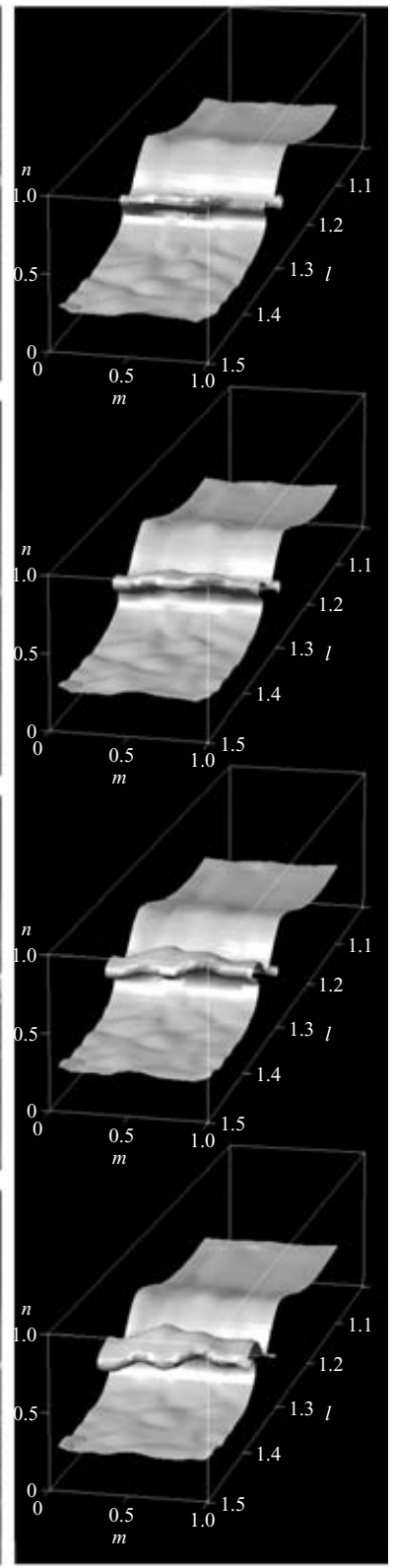

FIGURE 7. UV light-induced fluorescence video images of the rebounding jet during a laboratory experiment $((a)$ perspective side view, $(b)$ wave-face view), and $(c)$ corresponding shapes of a plunging jet in a computation for case 2 . The time-interval for both results is $0.032 \mathrm{~s}$. The experiment was conducted in a wave flume $8 \mathrm{~m}$ long, $0.25 \mathrm{~m}$ wide and $0.5 \mathrm{~m}$ high with a $1: 20$ slope, equivalent to the geometry in our computing domain (cf. figure 1). The wave conditions were identical to case 2 , and the images were recorded by a high-speed video camera (recording frequency: $250 \mathrm{~Hz}$, resolution $640 \times 480$ pixels).

Peregrine (1983) identified some possible splash-up modes ('rebounds', 'intermediate' and 'pushes up'), after the plunging point. In our computations for plunging breakers, the presence of strong shear layers appears to depend upon the splash-up mode. 

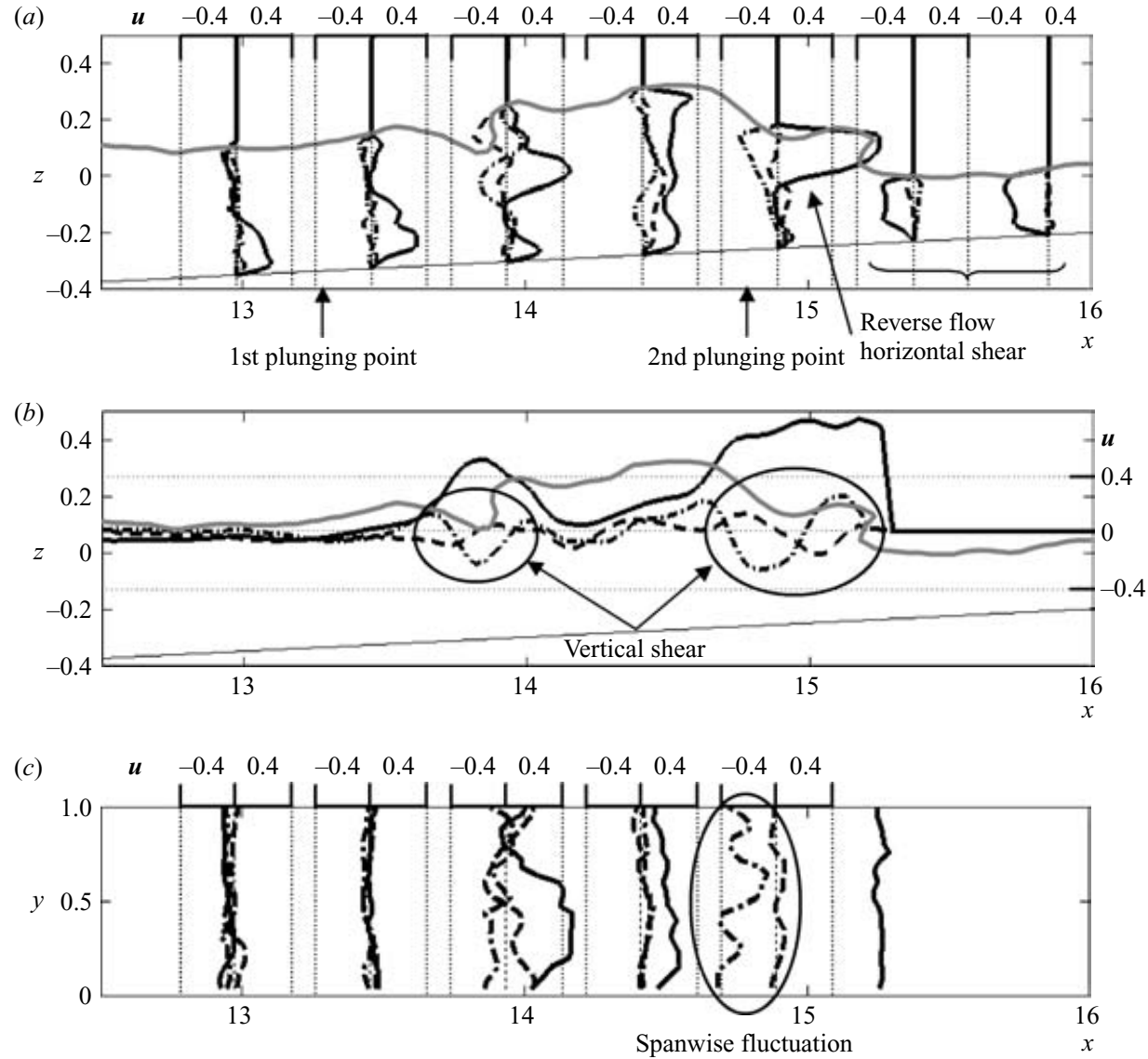

FIGURE 8. Instantaneous velocity distributions along $(a)$ the vertical axis at $y=0.5,(b)$ the horizontal axis at $y=0.5$, and $(c)$ the spanwise axis at $z=0.055$ at $t=t_{p}+2 T / 8$ for case 2 ; horizontal velocity (__ $)$, vertical velocity $(-\cdot-)$, spanwise velocity $(---)$, surface profile represented by grey lines.
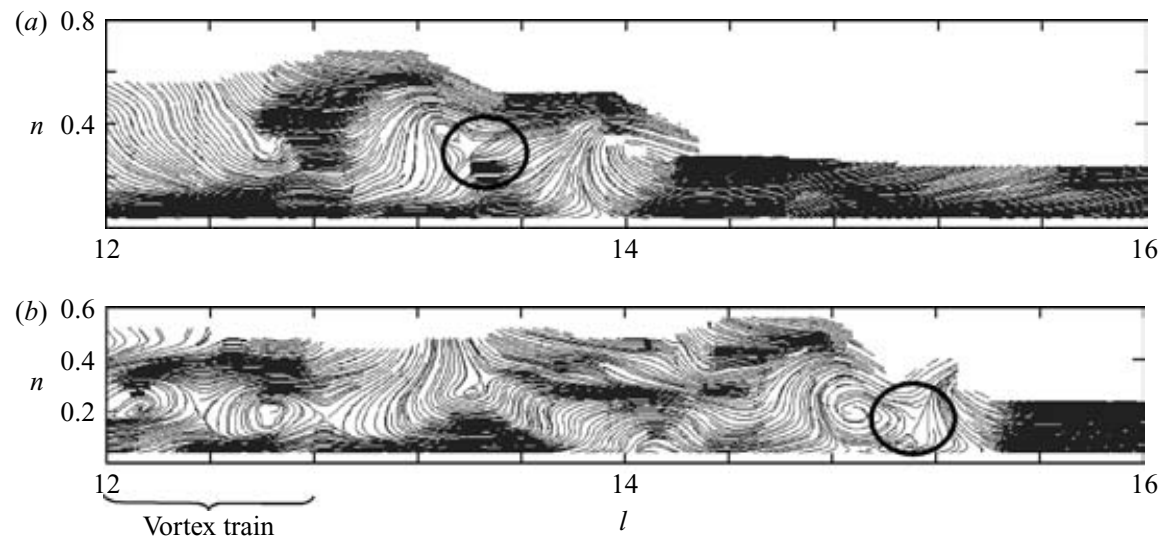

FiguRE 9. Streamlines at the centre of the computing domain $(m=0.5)$ during the splash-up cycle in case 2. (a) $t=t_{p}+T / 8$, (b) $t=t_{p}+2 T / 8$. 

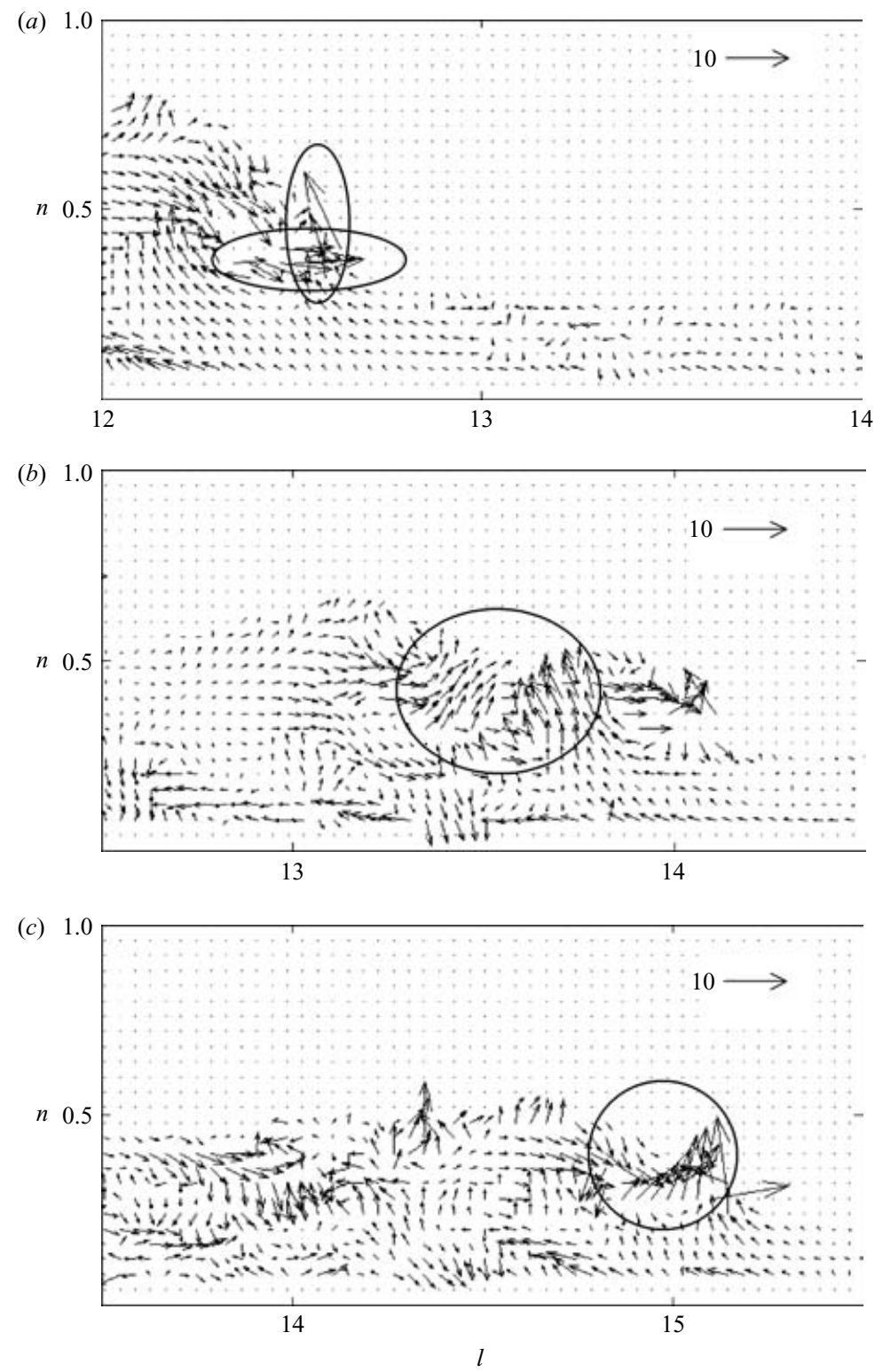

FIgURE 10. Principal axes and strain rate in case 2. (a) $t=t_{p},(b) t=t_{p}+T / 8$, (c) $t=t_{p}+2 T / 8$.

Figure 10 shows the principal axes and strain rates, corresponding to the eigenvectors and eigenvalues of the strain tensor, in the same cross-section as in figure 9. When the jet hits the wave face in the initial stage, a strong shear layer with horizontal stretching (corresponding to a high eigenvalue in a horizontal direction) is rapidly formed on the former surface beneath the jet (cf. figure 10a). High downward momentum following the jet then penetrates the forward water region to push it upward, forming a significant vertical shear layer in the pushes up mode. Very high eigenvalues in an obliquely upward direction occur on the shear layers behind the secondary jet pushed up in front of the plunging point, indicating that the fluid in this region is 
(a)

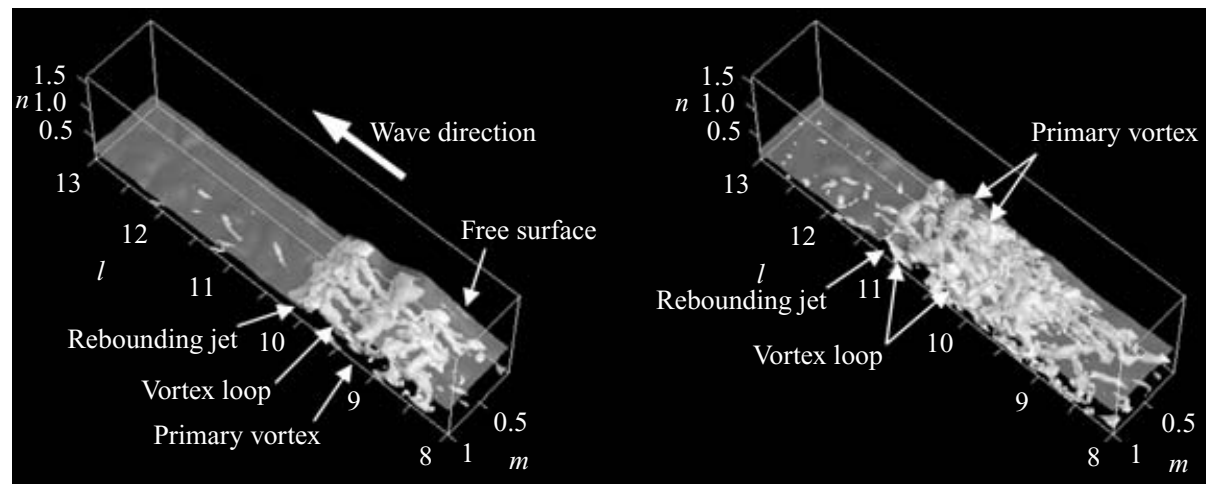

(b)

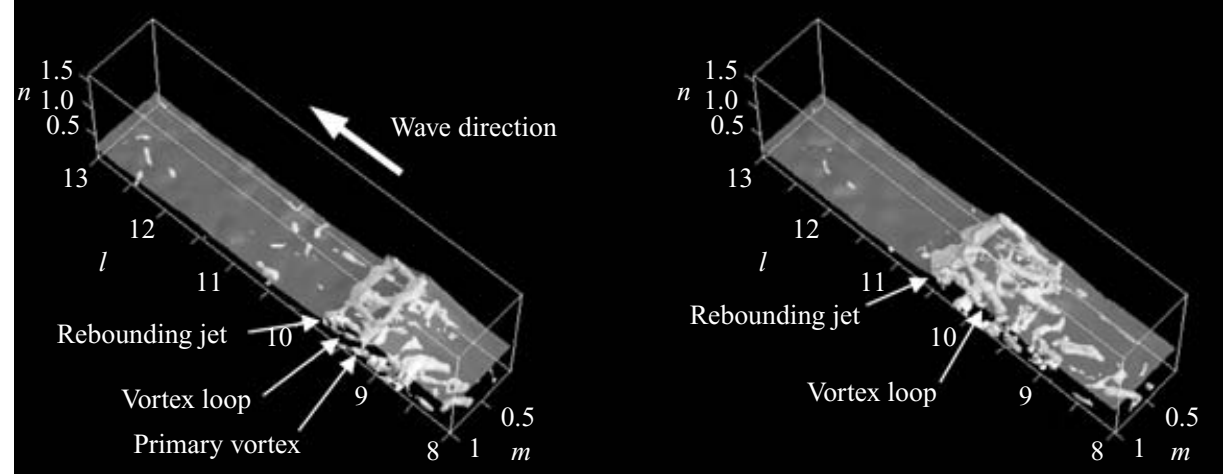

FIGURE 11. Distributions of vortex cores in a transition region in $(a)$ case 2 and $(b)$ case 4. Left-hand column, $t=t_{p}+T / 8$; right-hand column, $t=t_{p}+2 T / 8$.

significantly stretched in this direction (cf. figure $10 \mathrm{~b}, \mathrm{c}$ ). This region coincides with the saddle region in the shear produced by the secondary jet and the primary vortex behind it. The saddle-point flow exhibiting maximum stretch in the strain initiates the streamwise vorticity, yielding the counter-rotating vortices in the braid. This process is probably analogous to the three-dimensional instability of a plane shear layer (Lasheras \& Choi 1988), as discussed below.

\subsection{Coherent vortex formation}

We visualize the coherent vortices evolving in the inherent strain field under breaking waves in much the same manner as Andreassen et al. (1998). Figure 11 shows the distribution of vortex cores in the transition regions for cases 2 and 4 -i.e. isosurfaces of the negative second eigenvalue $\lambda_{2}$ of the tensor $L_{i j}=S_{i j}^{2}+\Omega_{i j}^{2}$, where $S_{i j}$ denotes the strain tensor and $\Omega_{i j}$ the vorticity tensor (cf. Jeong \& Hussain 1995). Longitudinal vortices with two hairpin-like bends in the saddle region are stretched on a plane sloping upward, and connect the upper part of the rebounding jet to the bottom part of the primary vortex (cf. also figure 12). At the inception of rebounding, the spanwise vorticity in the braid layer becomes unstable in the saddle region of the strain (cf. figure 10). The spanwise undulation of the vorticity is amplified by stretching along the eigenvector (i.e. the principal axis), causing a wavy vortex tube. Since both sides of the bends in the vortex tube have opposite vorticity, a selfinduction occurring between them displaces the down-stream bends (near the jet) upward and the up-stream bends downward, causing normal perturbations to the braid layer. Furthermore, at the down-stream bends, parts of the tube are pulled by 

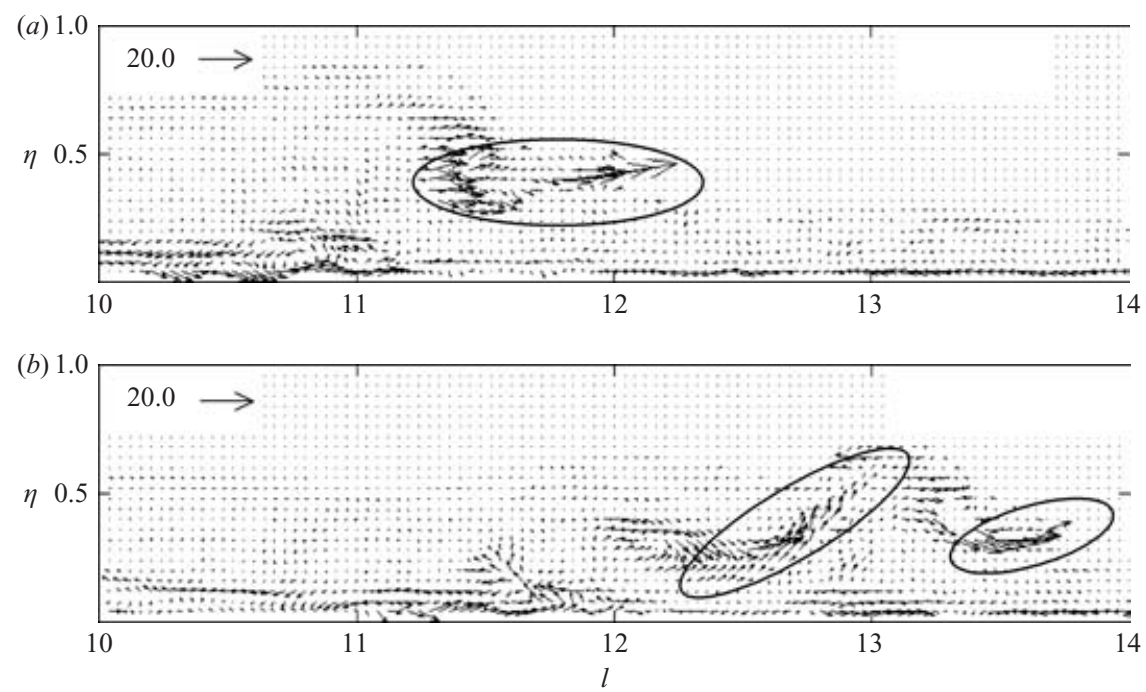

FIGURE 12 . Vorticity vectors in a transition region at the centre of the computing domain $(m=0.5)$ in case $5(a) t=t_{p} .(b) t=t_{p}+T / 8$.

the rotating forwarding jet, while the up-stream bends are wrapped by the subsequent primary vortex (cf. also figures 13 and 14). The stretched vortex tube intensifies its vorticity, and develop into a vortex loop with counter-rotating vorticity. While the next plunging stage yields a new primary vortex, further stretching and wrapping of the vortex loop organizes the so-called rib structure, involving the longitudinal (or rib) vortices enveloping the primary vortices (cf. figure 13 and also figure 18). The rib and primary vortices are subject to local stretching, and thus the vorticity and local strains are consecutively intensified even after the jets have passed, so that the vortex structure becomes complex with the vortices intertwined and entangled together. The rib structure was found in all of our simulations for plunging breakers, which suggests that the formation of this structure is a characteristic of the splash-up cycle for that breaker type.

On the other hand, the number of bends in the vortex loop tends to increase if the relative breaking wave height increases and the bottom slope steepens. Strongly plunging waves may yield longitudinally stretched vortex loops with three bends - i.e. three pairs of counter-rotating vorticity beneath the wave surface (cf. figures 14 and 15), rather than just two as in the simulations for cases 2 and 4 (cf. figures 11). In general, this occurs in very shallow water, where the vertical evolution of the vortices is inhibited by the bottom. The resulting vortex structure is packed within a shallowwater region under the descending surface after the breaking wave crest passes, and features a longitudinally elongated rib structure (cf. the cores at $t=t_{p}+2 T / 8$ in figure 15).

\subsection{Local surface deformation by subsurface vortices}

In addition to the water wave dynamic pressure, major factors defining transient local deformations of the surface include the vortices and vortex-induced pressure fluctuations beneath it. For instance, a vortex pair approaching a free surface from below pushes the surface up, and a nearby local depression (a 'scar') with a secondary vortex underneath may appear in low Weber number flows (Ohring \& Lugt 1991; Sarpkaya 1996). Brocchini \& Peregrine (2001a) summarize various aspects of surface 
(a) The downstream bends are pulled by the rebounding jet

The upstream bends are wrapped by the primary vortex

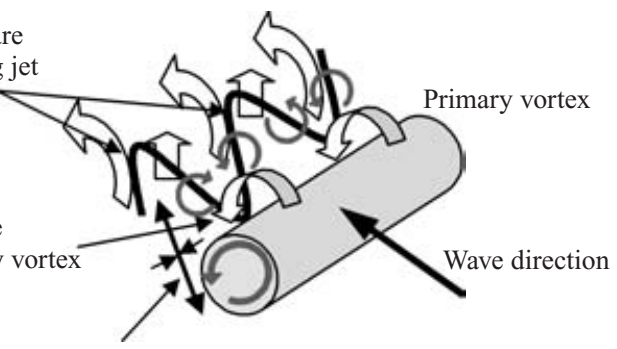

Stretch in the saddle region

(b)

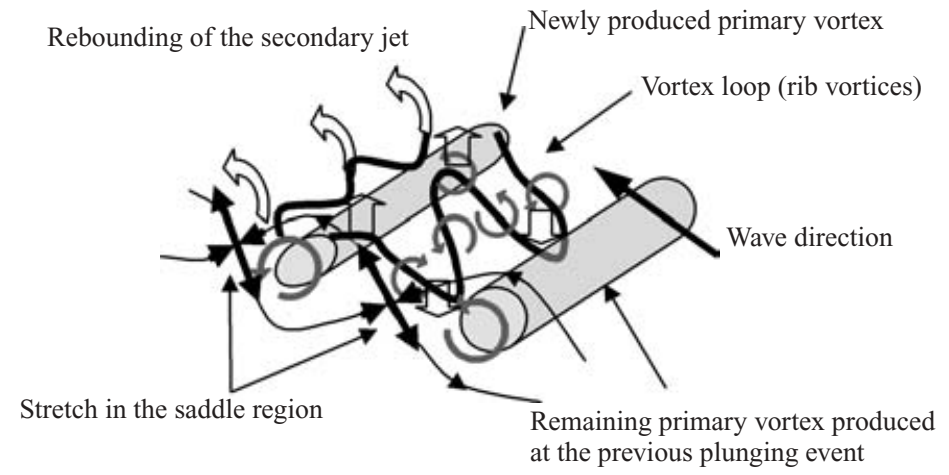

(c)

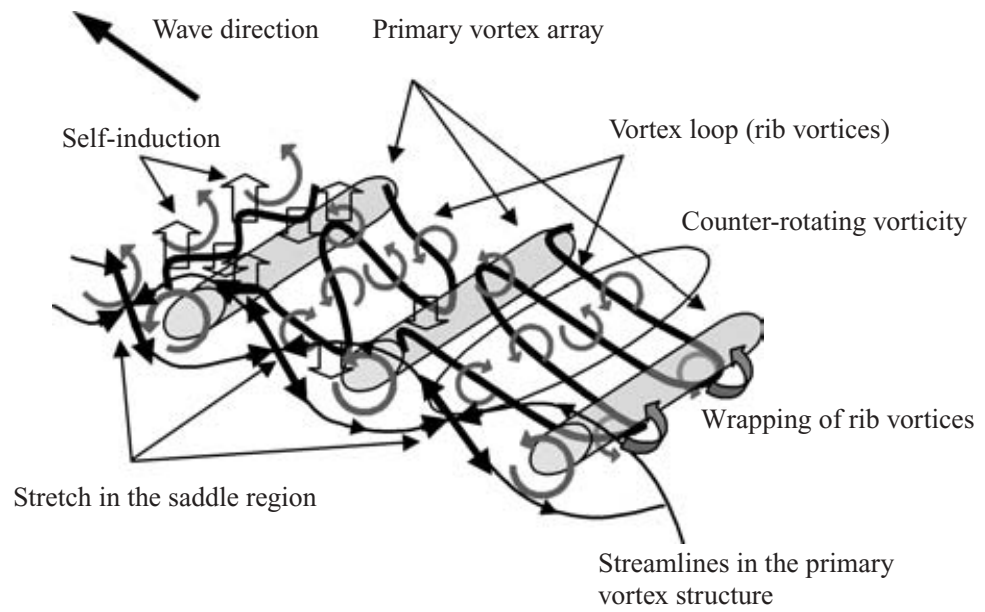

FIGURE 13. Schematic representation of the mechanism to form the vortex loops evolving into the rib structure (a) Initiation of the vortex loop at the first plunging point. (b) Consecutive formation of the vortex loops. $(c)$ Evolution of the rib vortices in a prinary vortex array.

deformation involving turbulence in their wide-ranging review, and in particular they envisage a physical mechanism involving a subsurface separation to generate scars in a typical vortex-induced deformation.

Figure 14 shows the distribution of vortex cores and simultaneous surface deformations at the second plunging phase for case 5. Several longitudinal depressions (scars) of the surface are generated on the jet, forming a rib-like deformed surface there. The rotating flow in the subsurface rib vortices can entrain fluid downward 
(a)
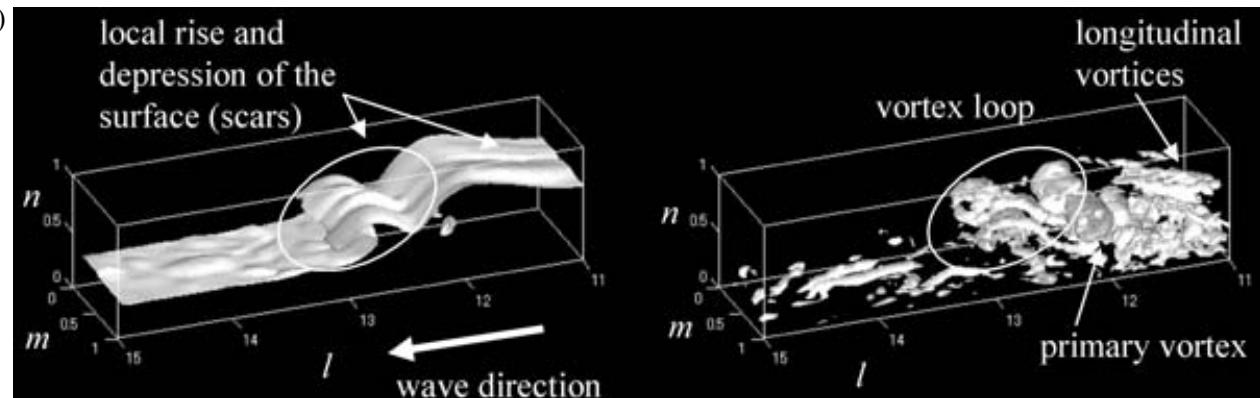

(b)

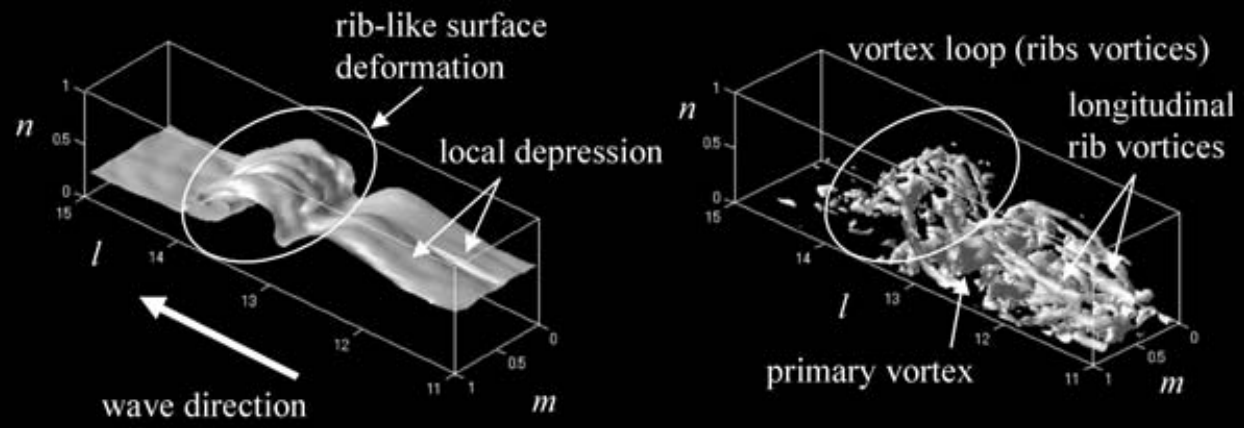

FIGURE 14. Shape of a free surface (left-hand column) and simultaneous vortex cores (right-hand column) in the secondary jet in case 5. (a) $t=t_{p}+9 T / 128 ;(b) t=t_{p}+18 T / 128$.

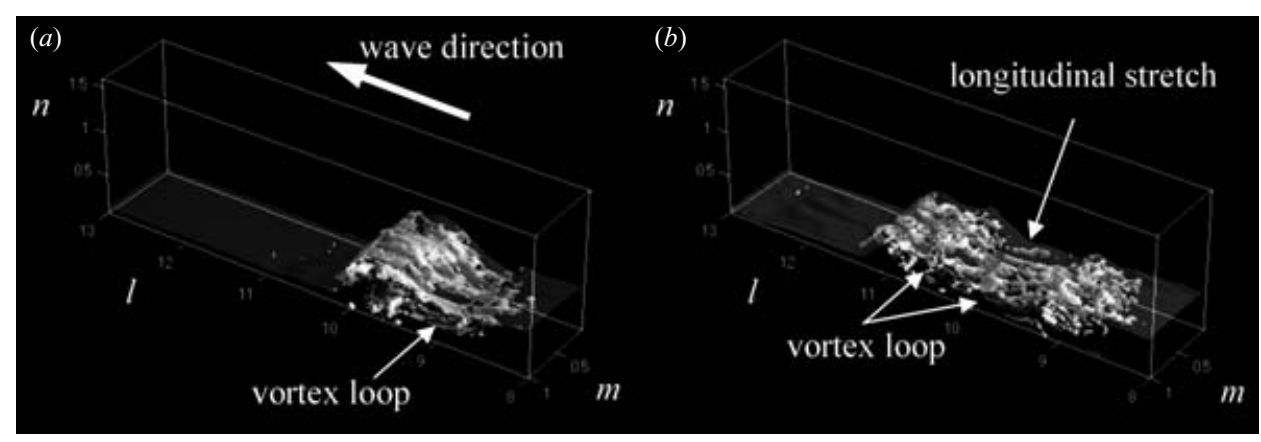

FiguRE 15. Distributions of the vortex cores at $(a) t=t_{p}+T / 8$ and $(b) t=t_{p}+2 T / 8$ in case 3 .

from the surface, to form scars - cf. again Brocchini \& Peregrine (2001a). In passing, let us note, however, that the dimensions of the scars may depend upon the surface tension, ignored in our computation. It can be seen that scars are generated over voids between rib vortices (the straighter parts of the vortex loops) aligned to a sloping braid beneath the surface in the secondary jet, and therefore the distribution of the ribs near the surface correlates with the local rise and depression of the surface. As previously mentioned, a finger-like surface pattern appears at the toe of the rebounding jets, in both the numerical and experimental results (cf. figure 7). Owing to the counterrotating vorticity, in the early formation stage of the secondary jet there is a spanwise pressure gradient beneath the surface, and rotational entrainments of the surface by the subsurface vortices and the resulting subsurface pressure undulations are 
(c)
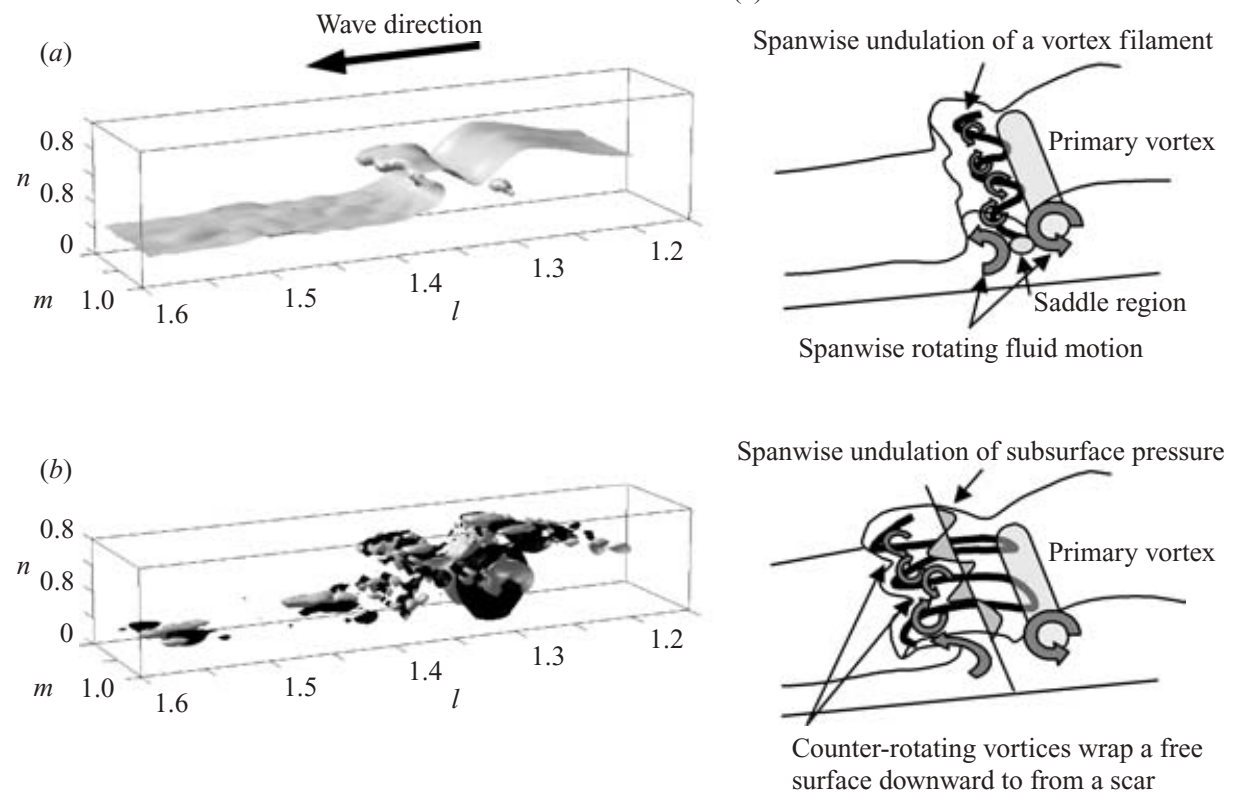

FIGURE 16. (a) Shape of the finger-like rebounding jet, $(b)$ simultaneous spanwise pressure gradient $\partial p / \partial m$ in the finger-shaped rebounding jet in case 2 (white surface, +0.01 ; black surface, -0.01$)$ and $(c)$ schematic representation of the mechanism to form the finger-shaped jet.

responsible for forming the finger-shaped jet (cf. also figure 16). The corresponding vortex-induced surface fluctuations also contribute to spanwise pressure gradient changes, generating transverse gravity waves.

\section{Obliquely descending eddies}

For a plunging breaker, the initial vortex loops stretch and align to a sloping shear layer, and evolve into rib vortices, as shown in the schematic representation of the mechanism in figure 13. These rib vortices are three-dimensional substructures consisting of ribs extending from the upper part of the forward primary vortex to the lower part of the rear primary vortex (the primary two-dimensional structure). Figure 17 shows a sequence of snapshots with bubbles in the obliquely descending eddies that we observed experimentally, for the wave conditions of case 2 (cf. also figure 8 in Nadaoka et al. 1989). We consider that the rib vortices (or stretched vortex loops) we find computationally correspond to these tilted columnar bubble clouds, and identify the obliquely descending eddies found by Nadaoka et al. (1989) with these rib vortices.

Nadaoka et al. (1989) showed that the principal axes of ensemble-averaged strains in a region behind a wave crest are inclined at about $45^{\circ}$ or less to the horizontal. They concluded that the mean strains are associated with evolution of the obliquely descending eddies, because the inclination of the principal axis of strain is analogous to the stretch direction of any obliquely descending eddy. Figure 18 illustrates transitions of the positions of the primary and rib vortices, described as projections of the vortex cores to the $(l, n)$-plane. The inclination angle of the $\mathrm{S}$-shaped rib vortex increases after the first plunging phase, and the maximum angle appears at the second plunging 
(a)

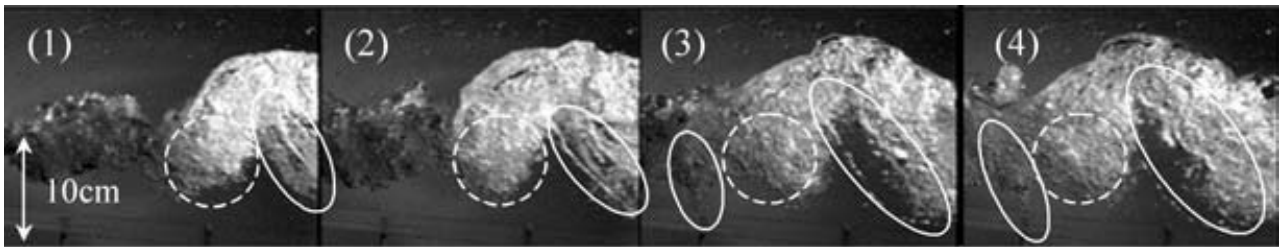

(b)

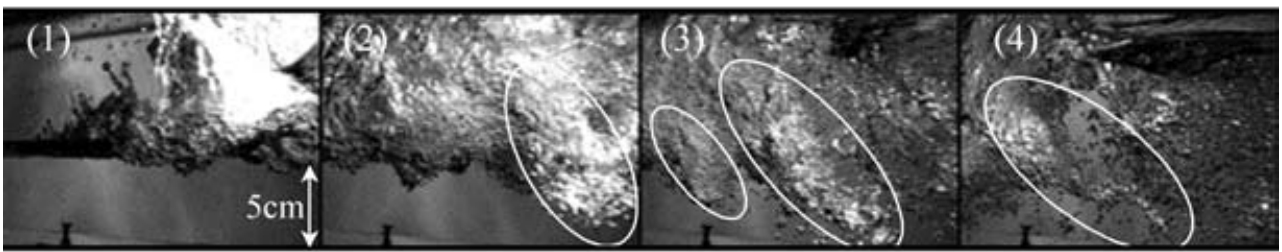

(c)

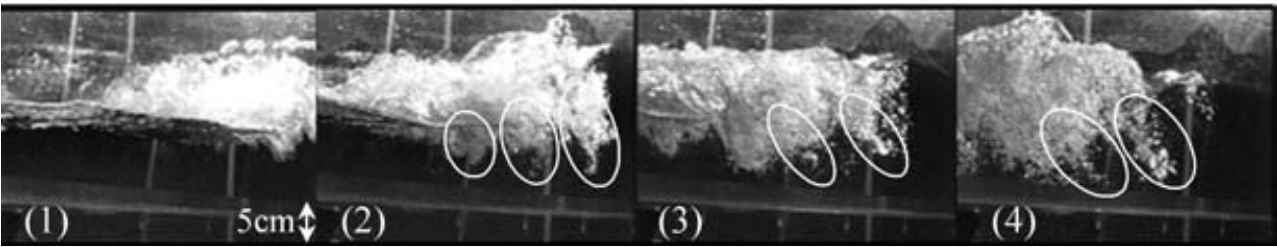

FIGURE 17. The bubble clouds formed in the surf zone in the wave conditions corresponding to case 2 during a visual experiment (solid circle: bubbles trapped in the obliquely descending eddy; broken circle: bubbles trapped in the primary vortex); $(a)$ side view of the bubble clouds at the second plunging point (time-interval: $0.04 \mathrm{~s}),(b)$ perspective rear view (time-interval: $0.08 \mathrm{~s}),(c)$ perspective front view (time-interval: $0.16 \mathrm{~s}$ ).
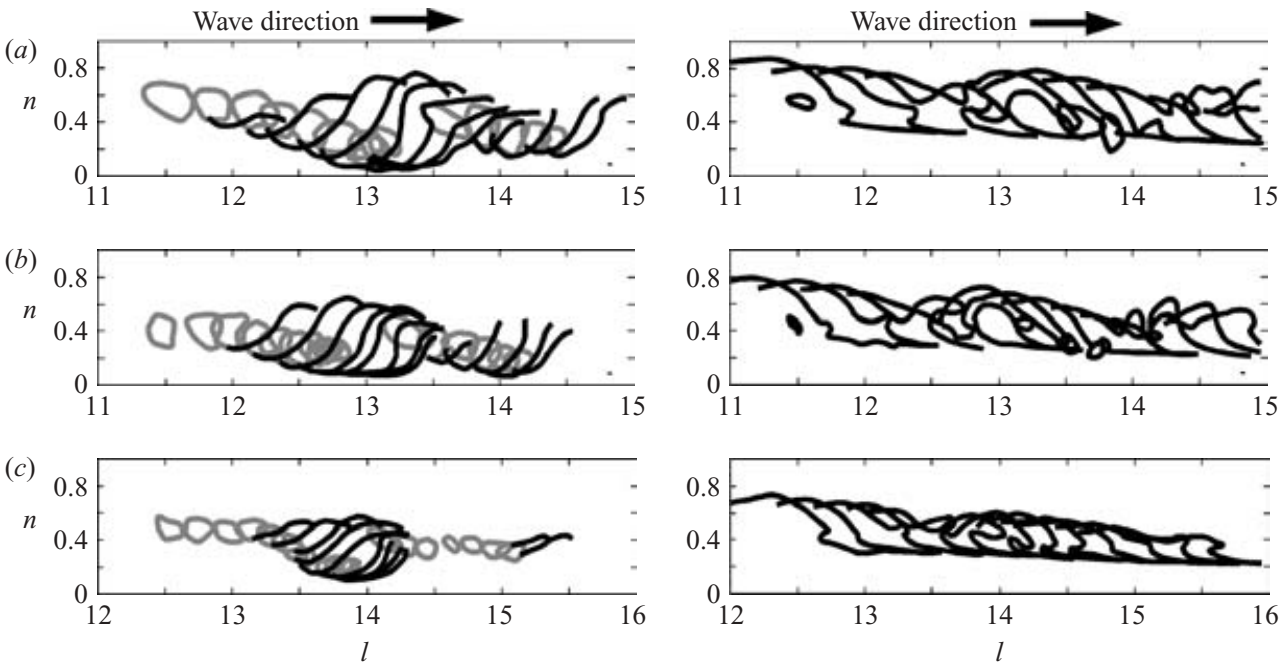

FIGURE 18. Evolution of the positions of the primary (represented by grey lines) and rib (black lines) vortices, and simultaneous wave shape for $(a)$ case $5,(b)$ case 2 and $(c)$ case 4; time interval: $9 T / 512$.

phase. The rib vortex is then stretched longitudinally with a decreased angle. It appears that restriction of the vertical transport of the vortices by the bottom contributes to the alignment of the rib vortex with the bottom. After the second plunging phase, the angle of the rib vortex appears to coincide with a typical tilting feature of the 

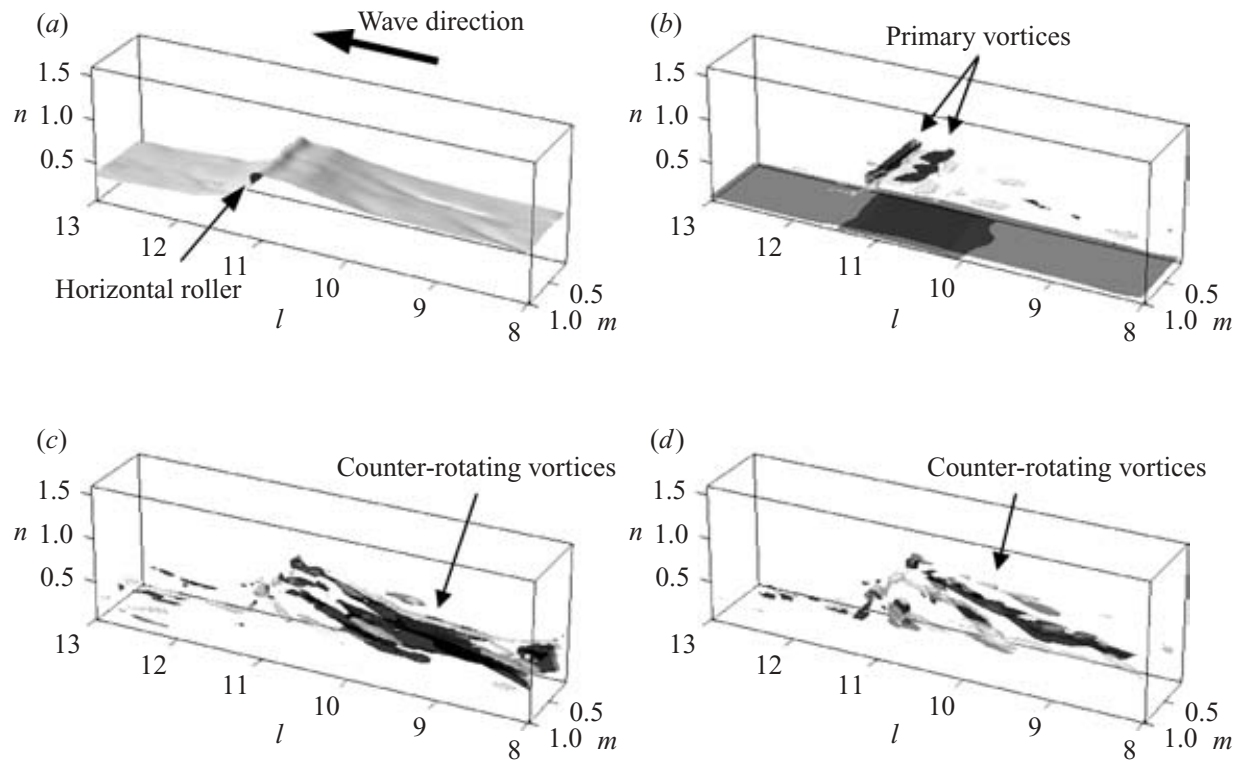

FIGURE 19. (a) Wave shape and simultaneous isosurfaces of $(b)$ spanwise, $(c)$ streamwise and (d) vertical vorticities at $t=t_{b}+3 T / 8$ in case 1 (white surface, +2.5 ; black surface, $-2.5 ; t_{b}$, wave breaking phase).

obliquely descending eddy observed over time (cf. figure 2 in Longo et al. 2002, and our figure 17). The maximum angle of the rib vortex may depend on the rebound height of the jet and the relative position of the primary vortex.

In contrast to a plunging breaker, where the vortices are rather closely packed, a spilling breaker typically occurs in deeper water. Thus, its vortices develop more freely, without significant restriction by the bottom, and they are also sparser so that there is less interaction between them. Figure 19 shows the wave shape and vorticity isosurfaces for case 1 . Several counter-rotating vortices, formed between the forward spanwise vortex in a horizontal roller and the subsequent vortex with the same rotation, are stretched longitudinally and trail from the vicinity of the breaking point. Whereas a plunging breaker is characterized by the cyclic plunging process of large jets, the relatively smaller jets in a spilling breaker produce a much weaker primary roller vortex. Spanwise vortex arrays, similar to those in a plunging breaker, but with much smaller length scales, form successively at a certain spacing throughout the process - and the maximum stretch primarily arises in the saddle region between them (cf. figure 20). Figure 21 shows the three-dimensional vorticity vectors beneath the free surface behind the wave crest. While the vortex vector demonstrates the existence of spanwise vortex filaments aligned with the crest, the filaments become unstable in the saddle region, and the undulation of the filaments is then amplified behind it. Vortex loops with counter-rotating vorticities are initiated in this process, and evolve into longitudinal vortices stretched obliquely downward (the obliquely descending eddies).

Figure 22 shows vortex core distributions under spilling breakers for case 1, and the evolution of the relative positions of primary rollers and longitudinal vortices. Longitudinal vortices are gradually intensified through consecutive stretching, and consequently a coherent longitudinal vortex structure predominantes, rather than the primary two-dimensional structure involving the primary spanwise vortex array in the initial stage of a plunging breaker. A side view of vortex cores (cf. figure 22) clearly 

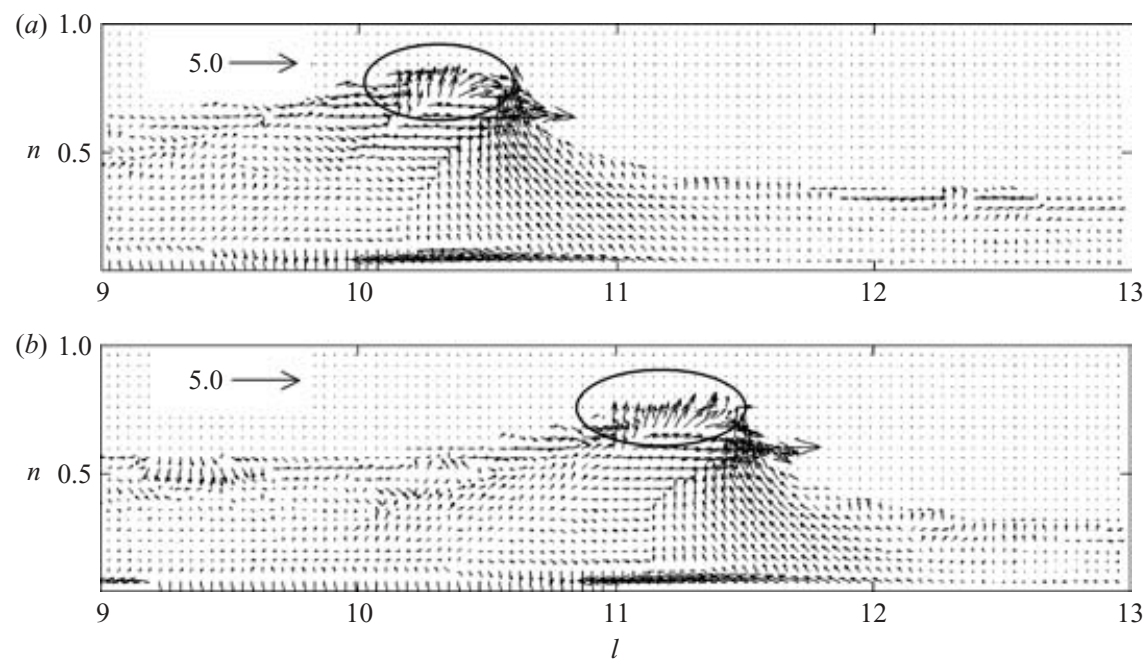

FIgURE 20. Principal axes and strain rate under a spilling breaker (case 1). (a) $t=t_{b}+2 T / 8,(b) t=t_{b}+3 T / 8$.

shows the obliquely descending vortex axis behind the crest, demonstrating that the simulated longitudinal vortex is again identical with a obliquely descending eddy. The inclination angles of the obliquely descending eddies were found to be smaller than in plunging breakers, which suggests that the evolution of the obliquely descending eddies is breaker dependent.

Figure 23 shows the trajectories of passive markers in an obliquely descending eddy. The markers released at the place circled in figure $22(d)$, when the wave crest passes above it, are initially advected by wave motion. They then encounter and are caught up by the obliquely descending eddy (cf. also figure 22c), when a typical spiral motion can be seen in the vortex. The radius of rotation gradually decreases as the angular velocity increases with time, which is typical of fluid motion in a vortex tube undergoing the stretch-and-intensification process. While the markers consecutively exhibit nearby spiral rotation, no mean transportation along the vortex axis has been detected in the simulation. In figure 10 of Nadaoka et al. (1989) and also our figure 22(c), the obliquely descending eddy has a typical oblique-downward vortex axis, although the marker trajectories appear to have a different orientation. While an upper part of the obliquely descending eddy is transported with the wave crest, a lower part tends to remain stationary behind it, since the mean velocity in transporting the vortices in this region is always less than that near the crest in the shear flow (cf. figure 24). Thus, the orientation of the vortex (corresponding to the angle of the obliquely descending eddy) and its vorticity varies with the progress of the breaking wave (cf. figure 22c). Thus, the marker trajectories are locally altered by the time-dependent orientation and local displacement of the obliquely descending eddy.

\subsection{Formation of bubble clouds in the obliquely descending eddies}

The presence of the obliquely descending eddy was identified by Nadaoka et al. (1989) from observation of the bubble clouds formed along the eddy (cf. figure 17). A physical mechanism for bubble trapping within a vortex was described by Tooby, Wick \& Isaacs (1977). They consider that fluid drag and gravity-buoyancy principally 

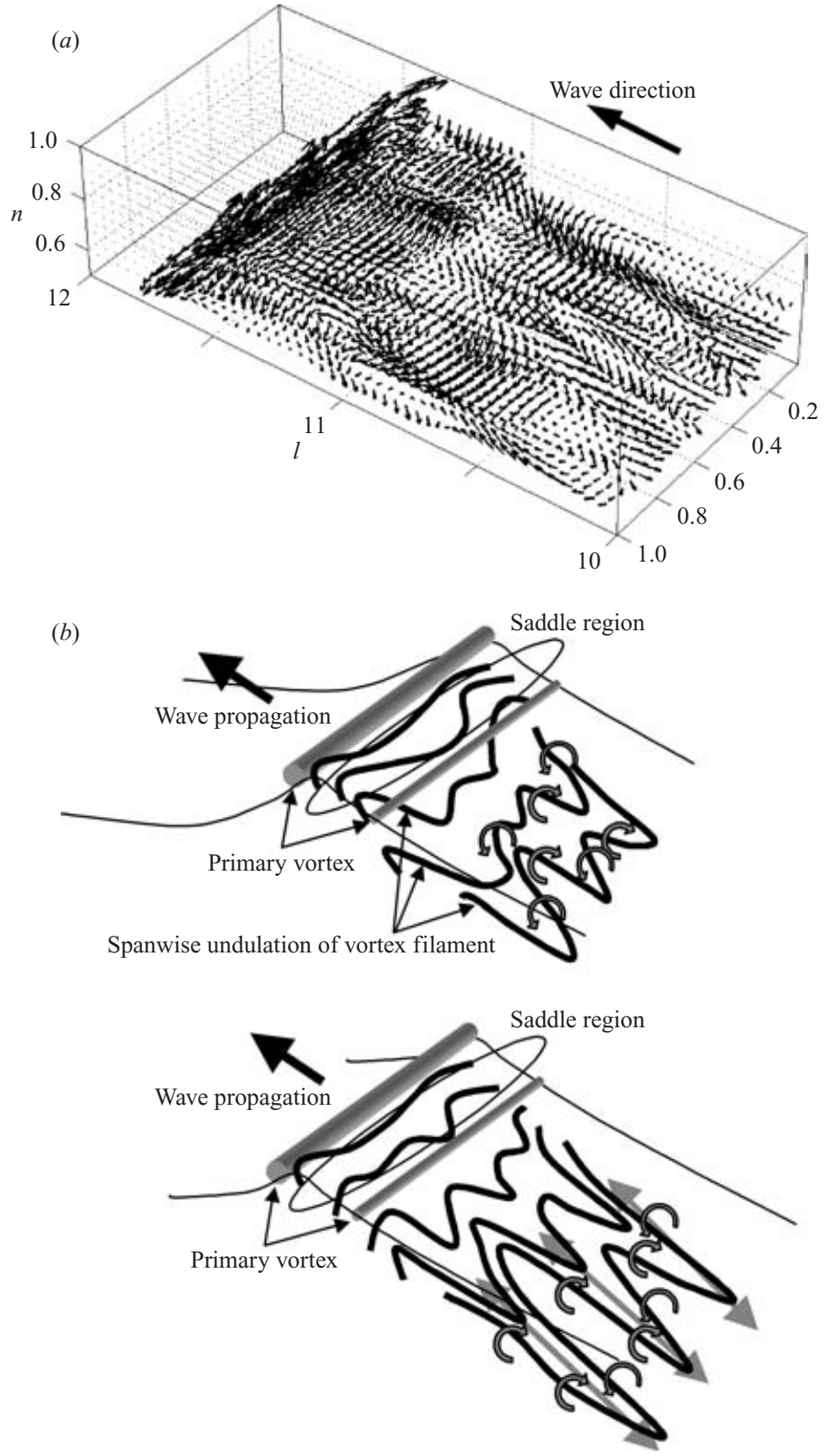

FigURE 21. ( $a$ ) Vorticity vector behind the wave crest at $t=t_{b}+3 T / 8$ and $(b)$ schematic representation of the evolution of vortex filaments. The undulations of the filaments are amplified in consecutive stretch, forming the obliquely descending eddies with the counter-rotating vorticity.

account for the formation of a circular orbit which a buoyant particle follows in rotating flow. Sene, Hunt \& Thomas (1994) discussed the role of an inertial effect (the radial pressure gradient) on bubble concentrations, and the formation of convergent trajectories in a coherent structure formed in a turbulent shear layer. Although the dynamic effect of bubbles was not included in our simulations, we speculate that the 

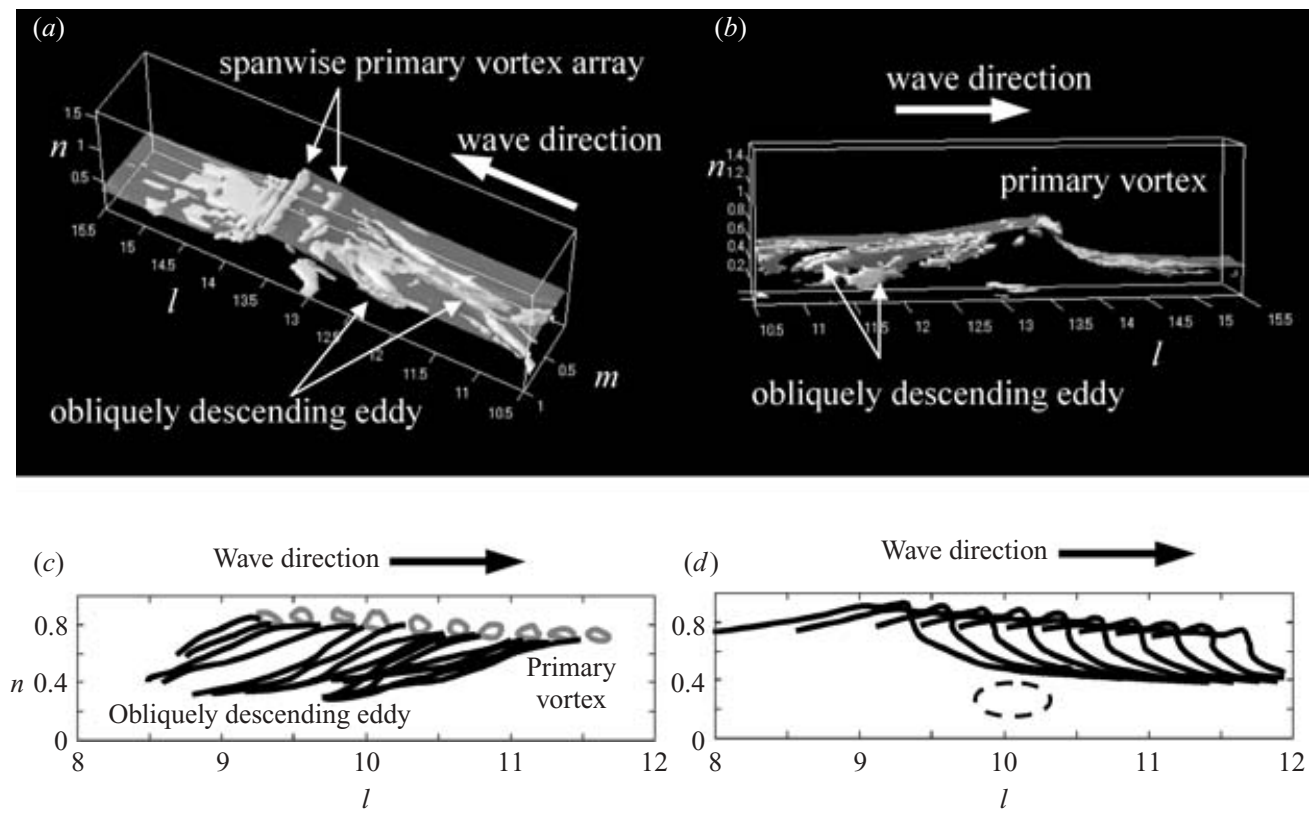

FIGURE 22. Distributions of the vortex cores $((a)$ perspective top rear view, $(b)$ side view) at $t=t_{p}+2 T / 8$, and evolution of $(c)$ the vortices and $(d)$ wave shape in case 1 (time interval: $9 T / 512)$.

inherent bubble cloud shown in figure 17 is probably caused by a trapping process as follows. First, bubbles entrained by plunging jets are transported to a certain depth by a downward flow in front of the primary vortex. Next, only those bubbles encountering the obliquely descending eddy with a certain streamwise vorticity are trapped within the vortex, because buoyancy locally balances the dynamic drag on the bubbles there (cf. figure 24) and the radial pressure gradient concentrates the bubbles near the vortex core where there is locally the lowest pressure. Thus, trapped bubbles remain within that part of the obliquely descending eddy where the radial pressure gradient in the streamwise vortex is high enough to prevent their buoyant motion for some time. Other more buoyant bubbles rise and rapidly disappear, leaving only the tilted columnar bubble clouds consisting of bubbles driven by the drag and inertial forces in spiral fluid motion, in the obliquely descending eddies (cf. again figure 17). Thus, only the upper part of an obliquely descending eddy, where the buoyancy and drag acting on bubbles can be in equilibrium, is visualized by the bubble clouds.

\section{Conclusions}

The formation of large-scale three-dimensional vortex structures in both plunging and spilling breakers has been simulated assuming gravity-dominated single-phase (liquid) flow without surface tension or strong surface distortions, or any dynamic modification of the vortex structures owing to aeration.

When an overturning jet projecting from the crest in a breaking wave rebounds from the free water surface ahead, the strain is locally intensified between the secondary jet and the primary spanwise vortex behind it. Apparent instability in the saddle region where the maximum stretch occurs almost instantaneously triggers a threedimensional velocity field at the plunging point. Thus, the spanwise undulation of the 

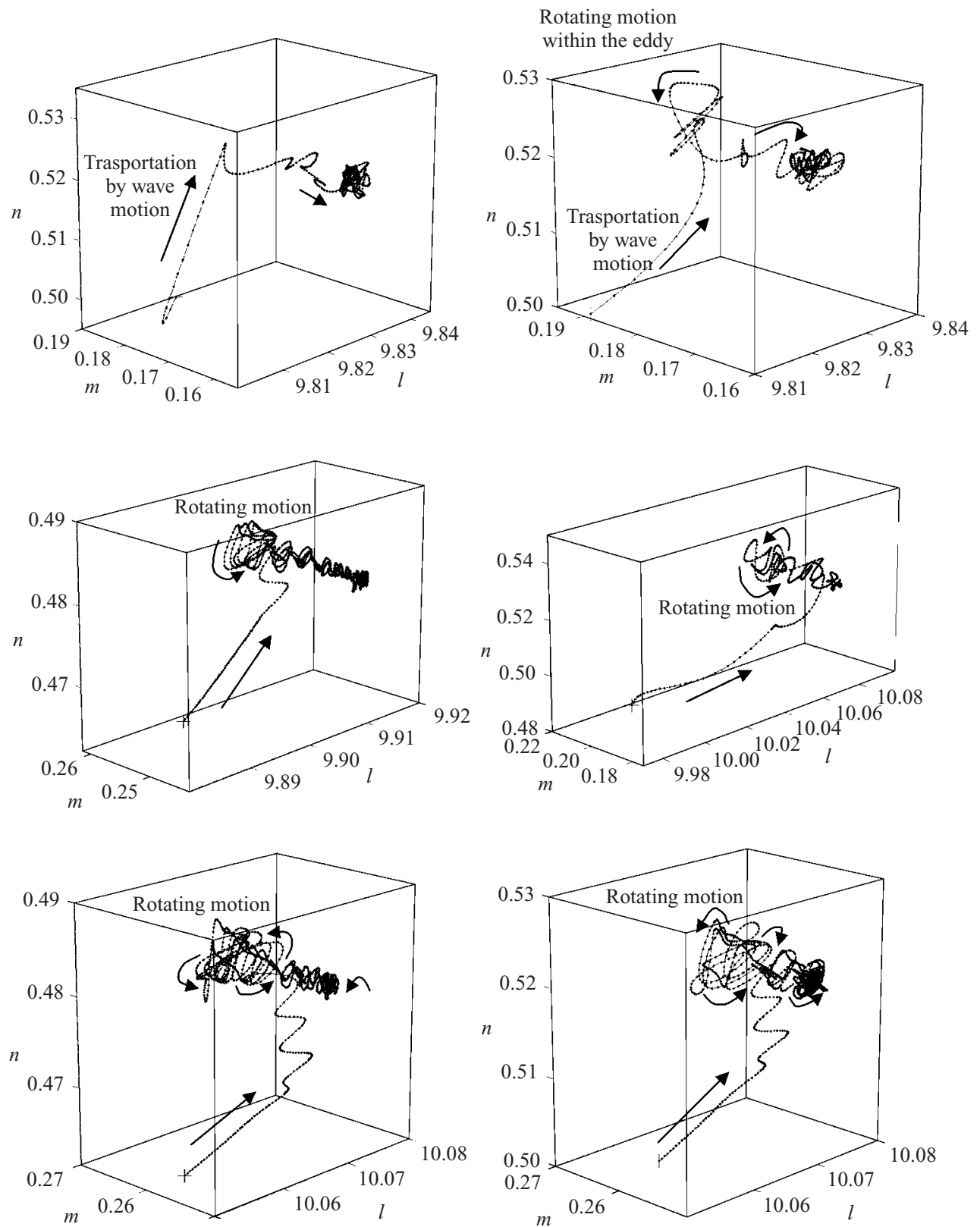

FIGURE 23. Typical trajectories of passive markers involved in the obliquely descending eddy in a spilling breaker; + , marker release point (the broken circle in figure $22 d$ is a release region for the markers).

vorticity, arising from re-orientation of perturbations in the primary vorticity, is first amplified in the saddle-point flow and leads to a wavy vortex loop. At the initial stage of the formation of the vortex loop, the spanwise undulation of vorticity produces a spanwise pressure gradient near the surface and wraps the surface around the vortex axis, to form a finger-shaped rebounding jet. A stretch-and-intensification process in the vortex loop along the principal axis of strain causes normal perturbations to a 

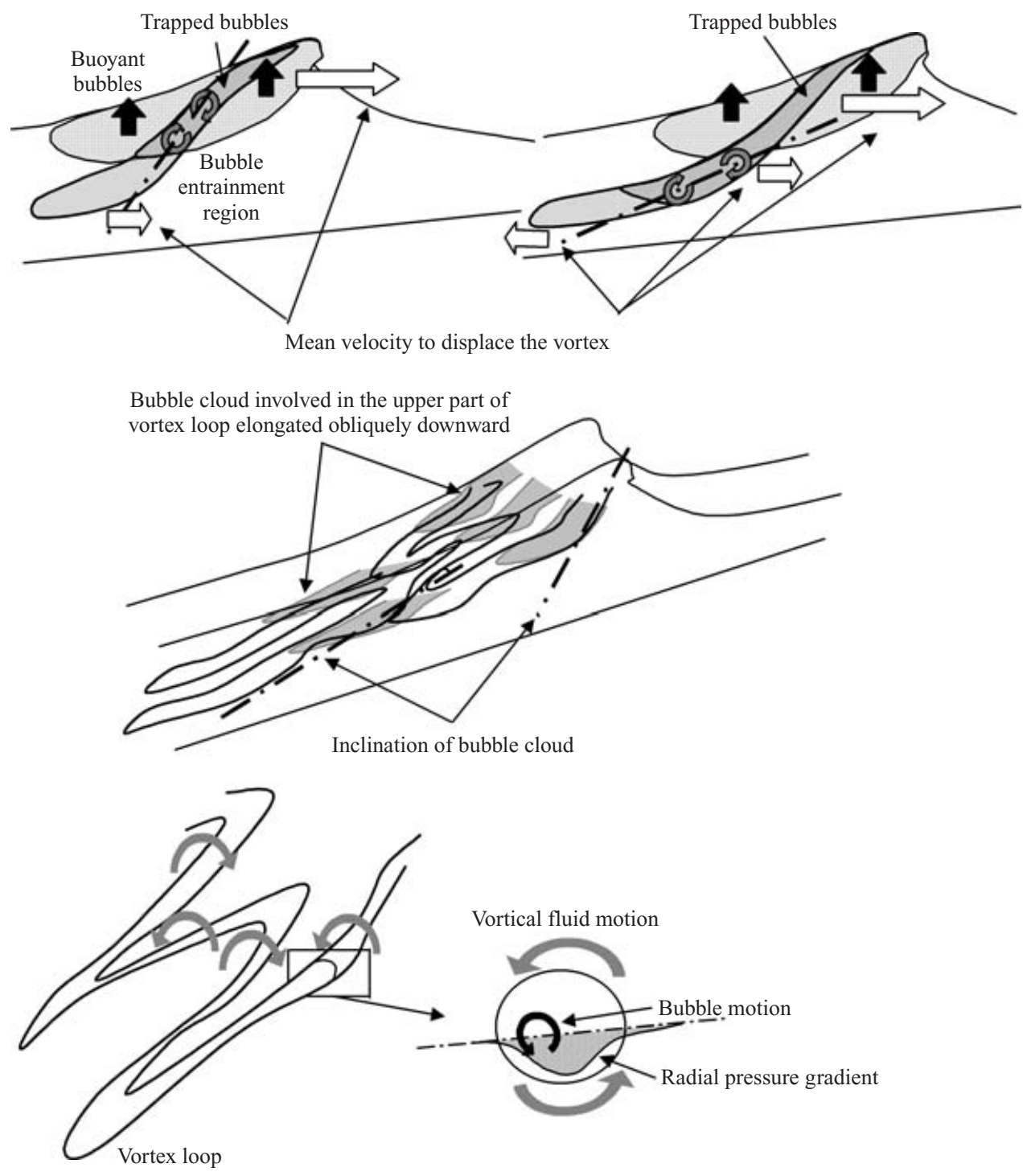

FIGURE 24. Schematic representation of the formation of columnar bubble clouds through bubble trapping within the vortex loop. The tilt angle of the obliquely descending eddy varies as a wave propagates because horizontal velocity to displace the eddy varies along the vortex axis.

braid layer, by self-induction between counter-rotating vorticities at the bends of the vortex loop. The continual stretching of the vortex loop in the braid organizes a rib structure, in which the vortex loop envelops the upper part of a new primary vortex produced by the next plunging jet to the bottom of the previous primary vortex behind it.

Continual deformation of the large-scale vortices and the production of new smallerscale vortices are caused by local strains intensified among the vortices, even after the breaking front has passed. While some of the vortices dissipate via a cascade (scaling-down) process in the shear field until the next breaking event, the remainder are transported in the reverse flow to the next wave breaking point and may trigger 
further instabilities, leading to a rather complex three-dimensional vortex structure. Thus, especially in the case of a plunging breaker in shallow water, a number of the large-scale vortices are packed within a shallow fluid region between the descending free surface and the bottom after the wave passes, which causes a more complex evolution of the vortex structure in the locally intensified strains between the closely packed vortices.

Horizontal shear flow under a broken wave, owing to shoreward flow in a progressing wave-front and reverse offshore flow beneath the wave trough level, can make the shear surface unstable - producing new spanwise vortices on the surface (cf. streamlines behind the plunging point in figure 9). Thus a vortex array, induced through $\mathrm{K}-\mathrm{H}$ instability on the plane shear surface, may develop into the so-called offshore vortex train found by Matsunaga et al. $(1988,1994)$. As the vortices remaining from the previous breaking event affect the formation of a new vortex structure via interaction among the vortices in the instantaneous field, the resulting aperiodic strains yield different vortex substructures over successive breaking events, resulting in varying turbulent flows at every breaking event. This variation may explain why intermittent turbulent flows occur at certain fixed points under successive breaking waves (cf. Cox \& Kobayashi 2000).

Obliquely descending eddies have been identified with the vortex loops (or rib vortices) stretched in the braid, which envelop adjacent primary two-dimensional vortices. In a plunging breaker, vortex loops that initially emerge in the saddle region are obliquely stretched from the bottom part of a primary vortex toward the upper part of a successive primary vortex formed at the next plunging phase, leading to the rib structure. The inclination angle of the obliquely decending eddy (the vortex loop) increases from the first to the second plunging phase, but decreases after that. The evolution of the obliquely descending eddy is evidently associated with transition of the wave shape, the rebounding height of the jet, and the spacing between the plunging points (or relative positions of the primary vortices). The rib vortices tend to lie longitudinally along on the bottom after the wave passes, since the bottom inhibits vertical evolution of the vortices. These longitudinal rib vortices may be those that Christensen \& Deigaard (2001) sometimes detected, instead of obliquely descending eddies.

In a spilling breaker, although consecutive plunges of its relatively smaller jets successively form an array of weaker primary vortices, three-dimensional instability occurs in the saddle region between its vortices in much the same way as for a plunging breaker. The spanwise vorticity undulation is amplified in this saddle region, and the resulting vortex loop is consecutively stretched in a sparser vortex field with less vorticity interaction, so that the obliquely descending eddy remains much longer than in a plunging breaker.

The trajectory of the fluid particles in the obliquely descending eddy typically exhibits vortex motion. Entrained bubbles, which are otherwise buoyant but happen to encounter an obliquely descending eddy, may be trapped within the vortex for a long time. The obliquely descending eddies tend to be displaced downward beneath the lowered surface behind the breaking wave, so trapped bubbles near the vortex cores may be taken to significant depths.

Financial support for this study was provided by JSPS Grants-in-Aid Scientific Research. R.J.H. was partly supported by a UBD Research Grant. The authors would also like to thank Professor Philip L.-F. Liu and an anonymous referee, for comments that led to the discussions in Appendix A and Appendix B, respectively. 


\section{Appendix A. Validation of the wave-breaking model}

The validity of the present wave-breaking model has previously been examined, with respect to the similarity of the computed breaking wave shape with experiment (Watanabe \& Saeki 1999). However, further validation is probably desirable to evaluate its accuracy in predicting wave-breaking turbulent flow, since surface shape similarity may not necessarily ensure that local turbulence is simulated adequately.

In order to deal with time-dependence in a sequence of breaking waves, several statistical definitions of turbulence have been proposed (cf. Petti \& Longo 2001). Turbulent velocity fluctuations, defined as deviations from phase averaging of the velocity, have been used in many other studies (e.g. Ting \& Kirby 1995, 1996; Cox \& Kobayashi 2000). Although efforts to reduce aeration effects on measured velocities may be made, the resulting statistics may nevertheless involve uncertainty associated with erroneous and drop-out signals due to bubbles, because strong turbulence typically occurs in highly aerated regions. Although these influences make it difficult to compare experiments and computation, a qualitative assessment can be made, provided that the related errors involved are statistically negligible.

Since a filtering operation is used in LES, the computational results must be processed appropriately in order to compare with experiment. We considered the corresponding turbulent energy in the same manner as Sagaut (2001). Thus, the instantaneous GS velocity $\overline{u_{i}}$ is defined to be

$$
\overline{u_{i}}=\left\langle\overline{u_{i}}\right\rangle+\overline{u_{i}},
$$

where the phase-averaged GS velocity is $\left\langle\overline{u_{i}}\right\rangle$, the fluctuation is ${\overline{u_{i}}}^{\prime}$. The phase-averaged exact solution $\left\langle u_{i}\right\rangle$ can be written as

$$
\left\langle u_{i}\right\rangle=\left\langle\overline{u_{i}}\right\rangle+\left\langle u_{i}^{*}\right\rangle,
$$

where $u_{i}^{*}$ is the SGS velocity. Since the exact velocity is written $u_{i}=\left\langle u_{i}\right\rangle+u_{i}^{\prime}$, the turbulent energy $k=\left\langle u_{k}^{\prime} u_{k}^{\prime}\right\rangle / 2$ derived from (A1) and (A2) is

$$
\frac{1}{2}\left\langle u_{k}^{\prime} u_{k}^{\prime}\right\rangle=\frac{1}{2}\left\langle\overline{u_{k}}{\overline{u_{k}}}^{\prime}\right\rangle+\langle q\rangle-\frac{1}{2}\left\langle u_{k}^{*}\right\rangle\left\langle u_{k}^{*}\right\rangle-\left\langle\overline{u_{k}}\right\rangle\left\langle u_{k}^{*}\right\rangle,
$$

where $q$ represents the SGS turbulent energy. Providing the phase-averaged SGS velocity $\left\langle u_{i}^{*}\right\rangle$ is much smaller than the other terms, we may neglect the third and fourth terms on the right-hand side of (A3) to obtain

$$
k \approx \frac{1}{2}\left\langle\overline{u_{k}}{\overline{u_{k}}}^{\prime}\right\rangle+\langle q\rangle .
$$

The phase-averaged velocity $\left\langle u_{i}\right\rangle$ is approximated as $\left\langle\overline{u_{i}}\right\rangle$ in this result.

The computed phase-averaged horizontal and vertical velocities and the turbulent energy in the transition region were compared with the experimental results of Ting \& Kirby (1995). While phase averaging was taken over 102 successive waves (or less when there is signal drop-out) for their experimental results, we considered only 30 quasi-steady waves because the computation is very time consuming. However, we believe this is sufficient to evaluate the turbulent velocity statistics, because the first- and second-order statistical moments of the velocity for samplings of 20, 25 and 30 waves were identical. Since Ting \& Kirby (1995) estimated the turbulent energy using a two-component laser-Doppler anemometer (LDA) on the basis of $k^{\prime}=1.33 / 2\left(\left\langle u_{1}^{\prime 2}\right\rangle+\left\langle u_{3}^{\prime 2}\right\rangle\right)$ in the same manner as Svendsen (1987), we calculated $k^{\prime}$ by using only two GS velocity components and $\langle q\rangle$ in (A4). We estimated a phase lag between zero-up crossing phases of the computational and experimental surface elevations at the breaking point (there is an unknown synchronized phase in the 

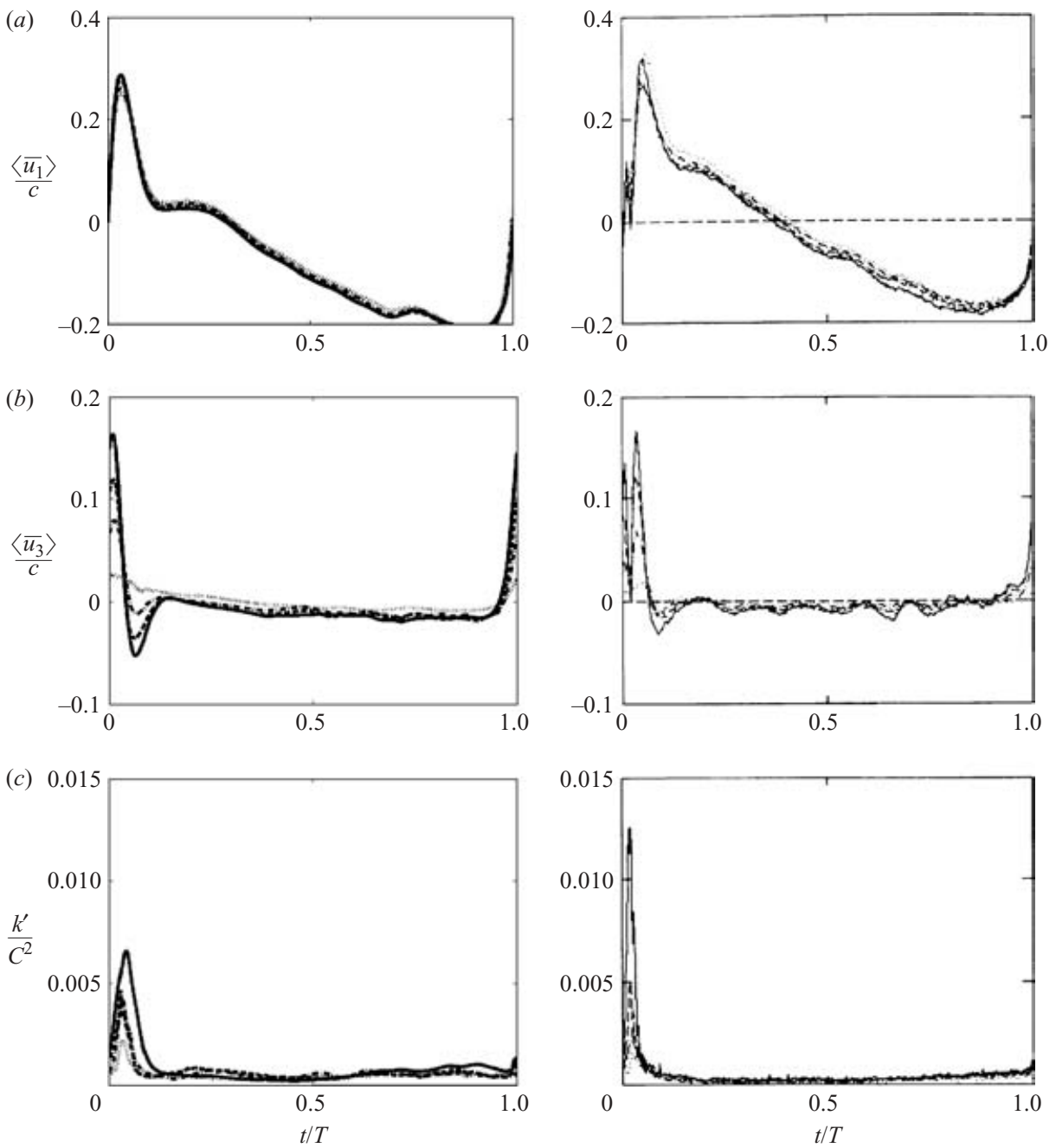

FiguRE 25. Numerical (left-hand) and experimental (right-hand) results of the phase averaged $(a)$ horizontal and $(b)$ vertical velocities, and $(c)$ the turbulent energy at $h / h_{b}=0.929$; $(z-\bar{\zeta}) / h=-0.2867(-),-0.4965(---),-0.7063(-\cdot-),-0.9161(\cdots)$, where $h$ is the local mean water depth, $h_{b}$ is $h$ at the breaking point and $\bar{\zeta}$ is the mean water surface elevation (wave set-up). [After Ting \& Kirby 1995].

experiment). The phase lag was commonly used to shift all of the time series at the different locations in the computation, in order to compare the experimental and computational results at the same phase. The computed $\left\langle\overline{u_{i}}\right\rangle$ and $k^{\prime}$ at the experimental measuring points were interpolated from neighbouring numerical grids. The modulus of the Jacobian elliptic function for an incident cnoidal wave was 0.999274, the bottom slope was $1 / 35$, the dimensionless breaking wave height and breaking water depth were 0.48 and 0.39 , respectively.

Figure 25 shows the phase-averaged horizontal and vertical velocities, and the turbulent energy at the relative depth $h / h_{b}=0.929$ ( $h$ is the local mean water depth at the measuring location; $h_{b}$ is $h$ at wave breaking) close to the first plunging point. While spiky fluctuations in $\left\langle u_{1}\right\rangle,\left\langle u_{3}\right\rangle$ and $k^{\prime}$ occur ahead of the maximum 

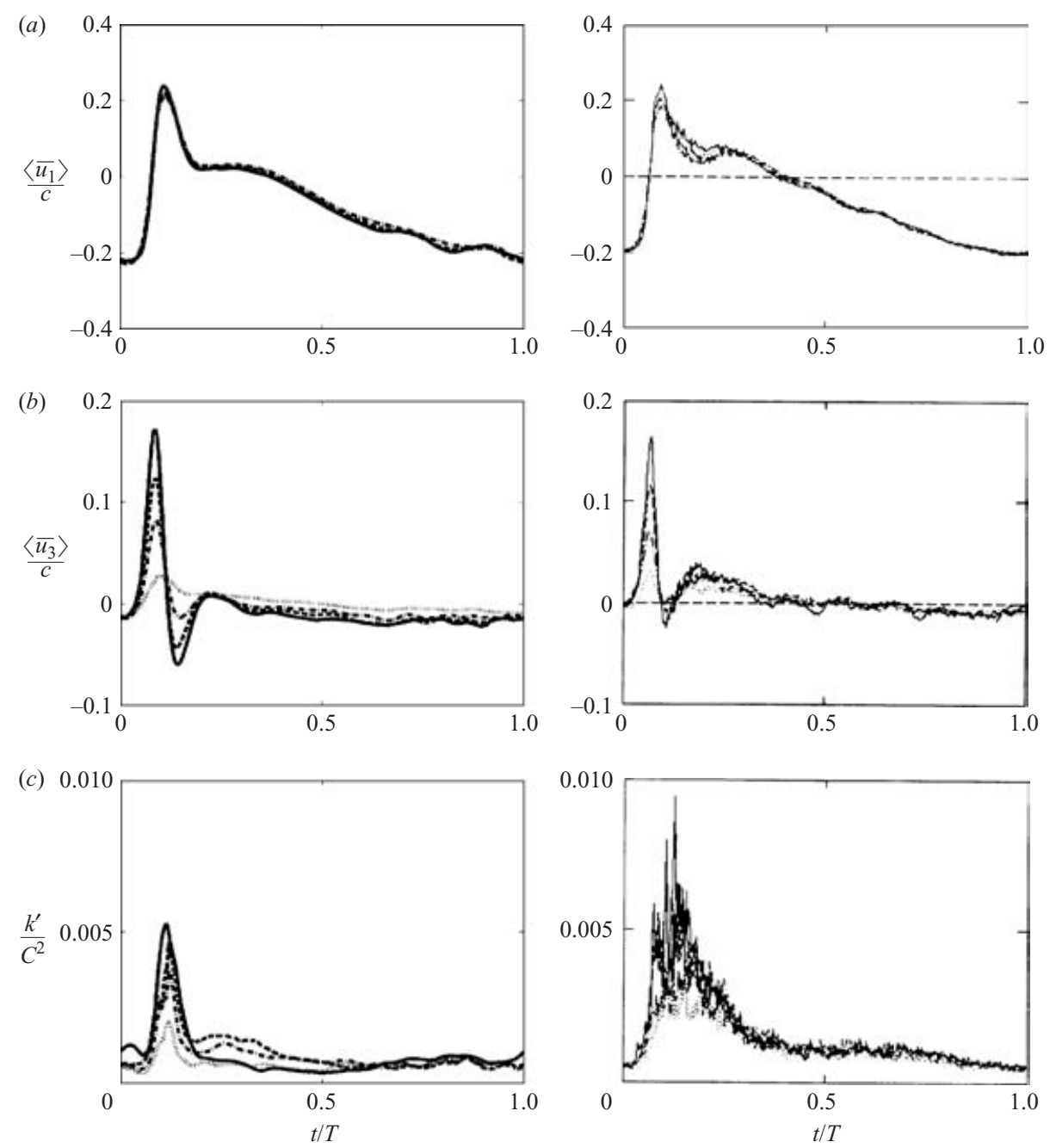

FiguRE 26. As for figure 25, but for values of $h / h_{b}=0.857 ;(z-\bar{\zeta}) / h=-0.4023(-$ ( $)$, $-0.5550(---),-0.7076(-\cdot-),-0.8603(\cdots)$. [After Ting \& Kirby 1995].

value of $\left\langle u_{1}\right\rangle$ in the experimental results, the computational results show smooth time series without such fluctuations. Figure 26 shows similar comparisons at $h / h_{b}=0.857$ (location between the first and second plunging points). It can be seen that the computed phase-averaged velocities have secondary crests similar to the experimental ones, although they appear to be somewhat lower. According to Ting \& Kirby (1995), the location where the water is pushed up by the plunging jet to form a new wave was aerated when the secondary crest appeared in the experiment.

Turbulent energy discrepancies in the phase-averaged velocity between the first and second crests suggest that the presence of a large number of bubbles may alter the statistics to some extent. There can be two possible effects in a liquid phase. One is the erroneous output signal induced at a vacuous measuring point, as mentioned above. Although an effort may be made to correct the erroneous signal by removing the signal drop-out, the consequent lack of data in strongly aerated regions cannot 
be overcome (Longo et al. 2002), so there is uncertainty in the turbulent statistics (especially in higher moments or higher-order statistics).

There can also be intensification of small-scale turbulence around bubbles, via wake effects in bubble-bubble interactions (Bunner \& Tryggvason 2002a). Bunner \& Tryggvason $(2002 b)$ found the turbulent energy induced by the fluctuating motion of buoyant bubbles increases with void fraction, and scales with the void fraction multiplied by the square of the average bubble rise velocity. It has also been found that the presence of bubbles in turbulent boundary layers intensifies the turbulent energy in proportion to the void fraction (Gabillet, Colin \& Fabre 2002).

In the context of breaking waves, the bubble concentration reaches $15-20 \%$ of the peak void fraction just after wave breaking (Cox \& Shin 2003), so the turbulent energy in initially highly aerated regions may indeed be intensified by small-scale fluctuations in the bubble motion owing to wake effects. The quantitative difference in the turbulent energy peaks between the computational and experimental results appears only at the closest measuring point to the surface, where there are probably aeration consequences for both (cf. the solid lines in figures $25 c$ and 26c). However, these spiky fluctuations in the turbulent energy have very short duration and small impulse (time integral of the spiky components), and they make a much smaller statistical contribution to the mean velocity than that due to the breaking wave over one wave period. Thus, the spiky fluctuations due to aeration are relatively unimportant compared with the shear turbulence due to the splashing jets, in the overall dynamics of the breaking waves. The computational results do show the major features in the phase-averaged velocities and the turbulent energy, so we do consider that our wave-breaking model adequately describes the flow field with relatively largescale variations after wave breaking, which is the main issue discussed in this context.

\section{Appendix B. Dynamic effects of bubbles on the vortex structures}

As discussed in $\S 4$, air bubbles appear entrained or maintained for a long time within the cores of the large-scale vortices generated by breaking waves (cf. figure 17). Thus, despite their buoyancy, bubbles are forced to spiral within the vortex cores owing to the water drag and pressure gradients (cf. figure 24). Nevertheless, it might seem that the dynamics of surf-zone breaking waves should be described as twophase (gas-liquid) flow with air bubbles of appropriate size distributions throughout the splash-up cycle - but it can be shown that this rather daunting prospect is unnecessary for understanding the major flows with relatively large-scale variations mainly affected by the gravity-dominated flow field. In this Appendix, we consider the extent to which bubbles may alter the local vorticity field in our computation, and consequently deform or displace the vortex structure (cf. Sridhar \& Katz 1999).

Let us consider an arbitrary volume $V$ of fluid bounded by its moving external fluid surface $S$ and a collection of moving internal surfaces $\left\{S_{B_{k}}, k=1, \ldots, N\right\}$ corresponding to $N$ relatively small bubbles that remain within $S$. The time derivative of the total momentum of this fluid volume is

$$
\frac{\mathrm{d}}{\mathrm{d} t} \int_{V} \rho \boldsymbol{v} \mathrm{d} \tau=\int_{V} \frac{\partial(\rho \boldsymbol{v})}{\partial t} \mathrm{~d} \tau+\int_{S} \rho \boldsymbol{v} \boldsymbol{v} \cdot \mathrm{d} \boldsymbol{S}+\sum_{k=1}^{N} \int_{S_{B_{k}}} \rho \boldsymbol{v} \boldsymbol{v} \cdot \mathrm{d} \boldsymbol{S},
$$

assuming no additional cavitation and hence inter alia that the normal component of the fluid velocity coincides with the surface velocity in the surface integrals. Moreover, these velocities coincide and are constant over each non-deforming bubble surface 
(and equal the particular bubble velocity), so that the bubble surface terms in (B1) are negligible for almost spherical bubbles on the Hinze scale. The equation of motion is simply

$$
\frac{\mathrm{d}}{\mathrm{d} t} \int_{V} \rho \boldsymbol{v} \mathrm{d} \tau=\boldsymbol{F}
$$

where $\boldsymbol{F}$ is the total force acting on the fluid volume $V$ (since all moving surface contributions here are zero). Treating the bubbles as 'mass-less' such that they do not contribute any (surface or body) forces, it follows that

$$
\int_{V} \frac{\partial(\rho \boldsymbol{v})}{\partial t} \mathrm{~d} \tau+\int_{S} \rho \boldsymbol{v} \boldsymbol{v} \cdot \mathrm{d} \boldsymbol{S}=\int_{S} \mathscr{T} \cdot \mathrm{d} \boldsymbol{S}+\int_{V_{T}} \rho \boldsymbol{g} \mathrm{d} \tau-\sum_{k=1}^{N} \int_{V_{B_{k}}} \rho \boldsymbol{g} \mathrm{d} \tau,
$$

where the terms on the right-hand side represent the surface and body forces due to the moving fluid $(\mathscr{T}$ denotes the stress tensor and $\boldsymbol{g}$ is the acceleration due to gravity, and $V_{T}=V+\sum_{k=1}^{N} V_{B_{k}}$ is the total volume of bubbly fluid under consideration). When there is a single bubble $\left(N=1\right.$ in $\left.V_{T}\right)$, this equation is identical to equation (A9) of Sridhar \& Katz (1999), although they considered a moving bubble inside a control volume with a fixed external boundary $S_{E}$ whereas in our context not only the bubble(s), but also the external fluid surface $S$, moves. It is again notable that the above equation differs from the corresponding case for single, phase fluid flow in two terms: the buoyancy forces on the bubbles (last term) and the boundary of integration in the first term (the bubbles move). Thus following Sridhar \& Katz (1999), the presence of a bubble alters the momentum by

$$
\Delta \boldsymbol{F}^{b}=\rho V_{B}\left\{\frac{\mathrm{D} \boldsymbol{v}^{0}}{\mathrm{D} t}-\boldsymbol{g}\right\},
$$

where $v^{0}$ is the undisturbed flow field at the location of any bubble if the bubble were absent, and $V_{B}$ is the bubble volume. Note that the additional momentum contribution corresponds to bubble motion relative to the moving external surface $S$ of volume $V$, therefore fully entrained bubbles (bubbles which are stationary relative to the moving volume $V$ ) do not contribute to $\Delta \boldsymbol{F}^{b}$.

Assuming the simplest case that identical-sized bubbles are preserved and uniformly distributed below the breaking waves in the surf zone, after filtering, equation (B4) becomes

$$
\begin{aligned}
\Delta \overline{\boldsymbol{F}_{\text {total }}^{b}} & =\int \sum_{k=1}^{N} G\left(\boldsymbol{x}-\boldsymbol{x}^{\prime}\right) \Delta \boldsymbol{F}_{i}^{b}\left(\boldsymbol{x}^{\prime}, t\right) \mathrm{d} \boldsymbol{x}^{\prime} \approx \int \rho N V_{B} G\left(\boldsymbol{x}-\boldsymbol{x}^{\prime}\right)\left\{\frac{\mathrm{D} \boldsymbol{v}^{0}\left(\boldsymbol{x}^{\prime}, t\right)}{\mathrm{D} t}-\boldsymbol{g}\right\} \mathrm{d} \boldsymbol{x}^{\prime} \\
& \equiv \rho \alpha V_{T}\left\{\frac{\mathrm{D} \overline{\boldsymbol{v}^{0}}}{\mathrm{D} t}-\boldsymbol{g}\right\},
\end{aligned}
$$

where $\alpha$ represents the void fraction.

When a wave first breaks, it is acoustically active inside the wave crest owing to newly created bubbles - with those smaller than the Hinze scale (about $1 \mathrm{~mm}$ ) subject to jet and drop entrainment, and larger bubbles fragmenting in the turbulent and sheared flow during the wave cavity collapse (Deane \& Stokes 2002). Subsequently, bubbles are no longer created during the much longer evolution of the newly formed bubble plume, which is therefore acoustically quiescent. The void fraction of air under deep-water breaking waves has been investigated by Lamarre \& Melville (1991), and in surf-zone breaking waves by Cox \& Shin (2003), and in general it is much less 

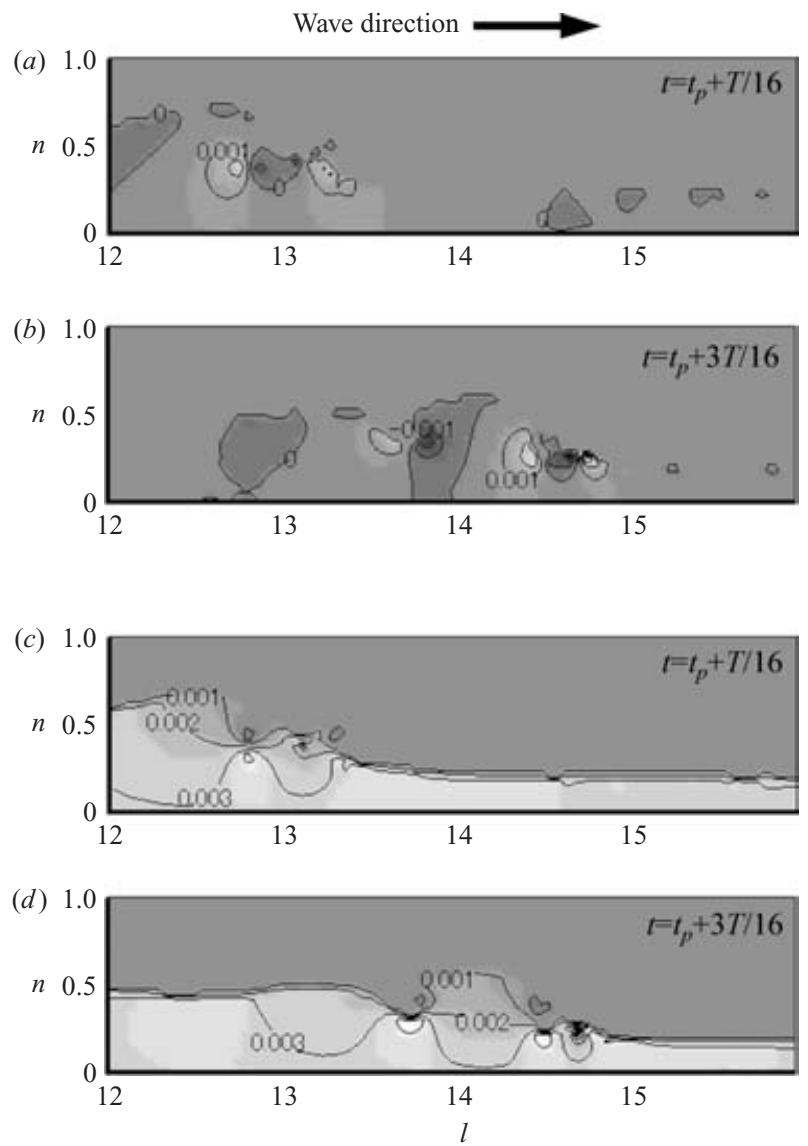

FiguRE 27. $(a, b)$ Horizontal and $(b, c)$ vertical displacements of fluid owing to the presence of bubbles at $20 \%$ void fraction in case 2 . (a) $t=t_{p}+T / 16$, (b) $t=t_{p}+3 T / 16$, (c) $t=t_{p}+T / 16$, (d) $t=t_{p}+3 T / 16$.

than the 15-20\% peak void fraction observed above the wave trough level. However, let us assume the void fraction throughout the surf zone is $20 \%$, so that the influence of bubbles on the dynamics of the vortex structure is not under-estimated. If larger bubbles formed during the collapse of the air pocket are provided, the Hinze scale may represent an appropriate scale of the bubble fragmented in sheared flow. Assuming bubble size at the Hinze scale, there are 48 bubbles in our computational cell volume.

The displacement of a fluid element in time $t$ is $a t^{2}$, where $a$ is the acceleration induced from (B2). Figure 27 shows the horizontal and vertical displacements due to the presence of bubbles during the characteristic phase interval $T / 8$ in case 2 . As Sridhar \& Katz (1999) also found, the buoyancy displaces the vortex core upward, whereas the force due to hydrodynamic pressure gradients reduces the core size and thus increases the vorticity. It can be seen that the primary vortices induce radial water displacement toward the vortex centre, causing a $0.4 \%$ reduction of the vortex core area. Assuming a steady state and ignoring viscous and turbulent diffusion during the time $T / 8$, a rough estimate based on the Helmholtz vortex theorem (the circulation $\Gamma=\oint_{C} \boldsymbol{u} \cdot \mathrm{d} \boldsymbol{x}=\int_{S} \boldsymbol{\omega} \cdot \boldsymbol{n} \mathrm{d} S$ in a vortex tube is preserved) is that the vorticity $\omega$ is 


$\begin{array}{cccccccc}\text { Case } & \overline{\delta_{x}} & \overline{\delta_{y}} & \overline{\delta_{z}} & \Delta_{\text {wave }} & \overline{\delta_{x}} / \Delta_{\text {wave }} & \overline{\delta_{y}} / \Delta_{\text {wave }} & \overline{\delta_{z}} / \Delta_{\text {wave }} \\ 1 & 0.0021 & 0.0011 & 0.0037 & 0.150 & 0.0140 & 0.0073 & 0.0247 \\ 2 & 0.0034 & 0.0015 & 0.0042 & 0.320 & 0.0106 & 0.0047 & 0.0131 \\ 4 & 0.0021 & 0.0003 & 0.0041 & 0.192 & 0.0109 & 0.0013 & 0.0214 \\ 5 & 0.0030 & 0.0011 & 0.0048 & 0.366 & 0.0082 & 0.0030 & 0.0131\end{array}$

TABLE 2. Relative displacements of a fluid element owing to bubbles and waves per $T / 8$.

intensified by only about $0.4 \%$, owing to the reduction of the cross-section of the vortex tube.

Table 2 shows displacements of fluid elements due to the presence of bubbles, approximate displacements due to a wave for $T / 8$, and the ratio between them for cases with the same bottom slope. $\overline{\delta_{x}}, \overline{\delta_{y}}$ and $\overline{\delta_{z}}$ denote mean values of the maximum streamwise, spanwise and vertical displacements, respectively. Assuming vortices under breaking waves are transported at the mean velocity of about $0.2 c$, where $c$ is the wave speed (Ting \& Kirby 1995; cf. also figures $25 a$ and $26 a$ ), the displacement due to a wave for $T / 8$ is roughly determined by $\Delta_{\text {wave }}=0.2 c T / 8$. Although the displacement due to buoyancy $\left(\overline{\delta_{z}}\right)$ is greater than that due to the pressure gradient in the vortex core $\left(\overline{\delta_{x}}\right)$, it is much smaller than the displacement of the primary vortices advected by the inertial force and dynamic pressure in the breaking wave. Consequently, the much faster generation and transformation of the vortex structures by the waves predominates, and the bubbles are unlikely to change the overall dynamics of the vortex structures significantly for the present cases - although they may significantly affect modulations of local turbulence. Further investigations on the evolution of the turbulence, and on the baroclinic generation of vorticity, are required to consider the energy and momentum transfer more generally throughout the breaking process.

\section{REFERENCES}

Andreassen, O., Hvidsten, P. O., Fritts, D. C. \& Arendt, S. 1998 Vorticity dynamics in a breaking internal gravity wave. Part 1 . Initial instability evolution. J. Fluid Mech. 367, 27-46.

Andreassen, O. \& Wasberg, C. E. 1994 Gravity wave breaking in two and three dimensions 1. Model description and comparison of two-dimensional evolution. J. Geophys. Res. 99, 8095-8108.

Ashurst, W. T. \& Meiburg, E. 1988 Three-dimensional shear layers via vortex dynamics. J. Fluid Mech. 189, 87-116.

Battues, J. A. 1988 Surf-zone Dynamics. Annu. Rev. Fluid Mech. 20, 257-293.

Brocchini, M. \& Peregrine, D. H. 2001a The dynamics of strong turbulence at free surfaces. Part 1. Description. J. Fluid Mech. 449, 225-254.

Brocchini, M. \& Peregrine, D. H. 2001b The dynamics of strong turbulence at free surfaces. Part 2. Free-surface boundary conditions. J. Fluid Mech. 449, 255-290.

Bunner, B. \& Tryggvason, G. $2002 a$ Dynamics of homogeneous bubbly flows. Part 1 . Rise velocity and microstructure of the bubbles. J. Fluid Mech. 466, 17-52.

Bunner, B. \& Tryggvason, G. $2002 b$ Dynamics of homogeneous bubbly flows. Part 2. Velocity fluctuations. J. Fluid Mech. 466, 53-84.

Chen, G., Kharif, C., Zaleski, S. \& Li, J. 1999 Two-dimensional Navier-Stokes simulation of breaking waves. Phys. Fluids 11, 121-133.

Christensen, E. D. \& DeigaArd, R. 2001 Large eddy simulation of breaking waves. Coastal Engng 42, 53-86.

Christensen, E. D., Walstra, D.-J. \& Emerat, N. 2002 Vertical variation of the flow across the surf zone. Coastal Engng 45, 169-198. 
Corcos, G. M. \& Lin, S. J. 1984 The mixing layer: deterministic models of a turbulent flow. Part 2. The origin of the three-dimensional motion. J. Fluid Mech. 139, 67-95.

Corcos, G. M. \& Sherman, F. S. 1984 The mixing layer: deterministic models of a turbulent flow. Part 1. Introduction and the two-dimensional flow. J. Fluid Mech. 139, 29-65.

Cox, D. T. \& ANDERson, S. L. 2001 Statics of intermittent surf zone turbulence and observation of large eddies using PIV. Coastal Engng J. 43, 121-131.

Cox, D. T. \& Kobayashi, N. 2000 Identification of intense, intermittent coherent motions under shoaling and breaking waves. J. Geophys. Res. 105, 14223-14236.

Cox, D. T. \& SHIN, S. 2003 Laboratory measurements of void fraction and turbulence in the bore region of surf zone waves. J. Engng Mech. 129, 1197-1205.

Deane, G. B. \& Stokes, M. D. 2002 Scale dependence of bubble creation mechanisms in breaking waves. Nature 418, 839-844.

Druzhinin, O. A. \& Elghobashi, S. 1998 Direct numerical simulations of bubble-laden turbulent flows using the two-fluid formulation. Phys. Fluids 10, 685-697.

Duncan, J. H. 2001 Spilling breakers. Annu. Rev. Fluid Mech. 33, 519-547.

FritTs, D. C., ARENDT, S. \& ANDRESSEN, O. 1998 Vorticity dynamics in a breaking internal gravity wave. Part 2. Vortex interactions and transition to turbulence. J. Fluid Mech. 367, 47-65.

Fritts, D. C., Garten, J. F. \& Andressen, O. 1996 Wave breaking and transition to turbulence in stratified shear flows. J. Atmos. Sci. 53, 1057-1085.

Gabillet, C., Colin, C. \& Fabre, J. 2002 Experimental study of bubble injection in a turbulent boundary layer. Intl J. Multiphase Flow 28, 553-578.

Gore, R. A. \& Crowe, C. T. 1989 Effect of particle size on modulating turbulent intensity. Intl J. Multiphase Flow 15, 279-285.

Hayakawa, M. \& Hussain, F. 1989 Three-dimensionality of organized structures in a plane turbulent wake. J. Fluid Mech. 206, 375-404.

Hirt, C. W. \& Nichols, B. D. 1981 Volume of fluid (VOF) method for the dynamics of free boundaries. J. Comput. Phys. 39, 201-225.

Jeong, J. \& Hussain, F. 1995 On the identification of a vortex. J. Fluid Mech. 285, 69-94.

Kolaini, A. R. \& Tulin M. P. 1995 Laboratory measurements of breaking inception and postbreaking dynamics of steep short-crested waves. Intl J. Offshore Polar Engng 5, 212-218.

Lamarre, E. \& Melville, W. K. 1991 Air entrainment and dissipation in breaking waves. Nature 351, 469-472.

Lasheras, J. C. \& ChOI, H. 1988 Three-dimensional instability of a plane free shear layer: an experimental study of the formation and evolution of streamwise vortices. J. Fluid Mech. 189, 53-86.

Li, L.\& Dalrymple, R. A. 1998 Instabilities of the undertow. J. Fluid Mech. 369, 175-190.

LiN, P. \& LiU, P. L.-F. 1998 A numerical study of breaking waves in the surf zone. J. Fluid Mech. 359, 239-264.

Lin, S. J. \& Corcos, G. M. 1984 The mixing layer: deterministic models of a turbulent flow. Part3. The effect of plane strain on the dynamics of streamwise vortices. J. Fluid Mech. 141, 139-178.

Liu, X. \& Duncan, J. H. 2003 The effects of surfactants on spilling breaking waves. Nature 421, $520-523$.

Longo, S., PetTI, M. \& Losada, I. J. 2002 Turbulence in the swash and surf zones: a review. Coastal Engng 45, 129-147.

Longuet-Higgins, M. 1995 On the disintegration of the jet in a plunging breaker, J. Phys. Oceanogr. 25, 2458-2462.

Matsunaga N., Takehara, K. \& Awaya, Y. 1988 Coherent eddies induced by breakers on a sloping bed. Proc. 21st Coastal Engng Conf. ASCE, pp. 234-245.

Matsunaga, N., Takehara, K. \& Awaya, Y. 1994 The offshore vortex train. J. Fluid Mech. 276, $113-124$.

Nadaoka, K., Hino, M. \& Koyano, Y. 1989 Structure of the turbulent flow field under breaking waves in the surf zone. J. Fluid Mech. 204, 359-387.

NAGAOSA, R. 1999 Numerical simulation of vortex structure and turbulent scalar transfer across a free surface in a fully developed turbulence. Phys. Fluids 11, 1581-1595.

OHRING, S. \& LugT, H. 1991 Interaction of a viscous vortex pair with a free surface. J. Fluid Mech. 227, 47-70.

Peregrine, D. H. 1983 Breaking waves on beaches, Annu. Rev. Fluid Mech. 15, 149-178. 
Petti, M. \& Longo, S. 2001 Turbulence experiments in the swash zone. Coastal Engng 43, 1-24.

Ruetsch, G. R. \& Meiburg, E. 1993 On the motion of small spherical bubbles in two-dimensional vortical flows. Phys. Fluids A 5, 2326-2341.

Sagaut P. 2001 Large Eddy Simulation for Incompressible Flows. Springer.

SarpKaya, T. 1996 Vorticity, free-surface, and surfactants. Annu. Rev. Fluid Mech. 28, 88-128.

SCARDOVELli, R. \& ZALESKI, S. 1999 Direct numerical simulation of free-surface and interfacial flow. Annu. Rev. Fluid Mech. 31, 567-603.

Sene, K. J., Hunt, J. C. R. \& Thomas, N. H. 1994 The role of coherent structures in bubble transport by turbulent shear flows. J. Fluid Mech. 259, 219-240.

Shen, L. \& Yue, D. K. P. 2001 Large-eddy simulation of free surface turbulence. J. Fluid Mech. 440, 75-116.

Shen, L., Zhang, X., Yue, D. K. P. \& Triantafyllou, G. S. 1999 The surface layer for free-surface turbulent flows. J. Fluid Mech. 386, 167-212.

SRIDHAR, G. \& KATZ, J. 1999 Effect of entrained bubbles on the structure of vortex rings. J. Fluid Mech. 397, 171-202.

Svendsen, I. A. 1987 Analysis of surf-zone turbulence. J. Geophys. Res. 92, 5115-5124.

Svendsen, I. A. \& Madsen, P. A. 1984 A turbulent bore on a beach. J. Fluid Mech. 148, 73-96.

Svendsen, I. A., Madsen, P. A. \& Hansen, J. B. 1978 Wave characteristics in the surf-zone. Proc. 16th Coastal Engng Conf. ASCE, pp. 520-539.

Ting, F. C. K. \& KirbY, J. T. 1995 Dynamics of surf-zone turbulence in a strong plunging breaker. Coastal Engng 24, 177-204.

Ting, F. C. K. \& Kirby, J. T. 1996 Dynamics of surf-zone turbulence in a spilling breaker. Coastal Engng 27, 131-160.

Toовy, P. F., Wick, G. L. \& IsaAcs, J. D. 1977 The motion of a small sphere in a rotating velocity field: a possible mechanism for suspending particles in turbulence. J. Geophys. Res. 82, 2096-2100.

Watanabe, Y. \& SAEKi, H. 1999 Three-dimensional large eddy simulation of breaking waves. Coastal Engng J. 41, 281-301.

Watanabe, Y. \& Saeki, H. 2002 Velocity field after wave breaking. Intl J. Numer. Mech. Fluids 39, 607-637.

Williamson, C. H. K. 1996 Three-dimensional wake transition. J. Fluid Mech. 328, 345-407.

YAкнот, V. \& OrszaG, S. A. 1986 Renormalization group analysis of turbulence (I. Basic Theory). J. Sci. Comput. 1, 3-51. 